\title{
Dependence Balance Based Outer Bounds for Gaussian Networks With Cooperation and Feedback
}

\author{
Ravi Tandon, Member, IEEE, and Sennur Ulukus, Member, IEEE
}

\begin{abstract}
We obtain new outer bounds on the capacity regions of the two-user multiple access channel with generalized feedback (MAC-GF) and the two-user interference channel with generalized feedback (IC-GF). These outer bounds are based on the idea of dependence balance which was proposed by Hekstra and Willems. To illustrate the usefulness of our outer bounds, we investigate three different channel models.

We first consider a Gaussian MAC with noisy feedback (MAC-NF), where transmitter $k, k=1,2$, receives a feedback $Y_{F_{k}}$, which is the channel output $Y$ corrupted with additive white Gaussian noise $Z_{k}$. For this channel model, the cut-set outer bound is not sensitive to the feedback noise variances. We demonstrate that our outer bound improves upon the cut-set bound for all nonzero values of the feedback noise variances. Moreover, in the limit as $\sigma_{Z_{k}}^{2} \rightarrow \infty, k=1,2$, our outer bound collapses to the capacity region of the Gaussian MAC without feedback.

Secondly, we investigate a Gaussian MAC with user-cooperation (MAC-UC), where each transmitter receives an additive white Gaussian noise corrupted version of the channel input of the other transmitter. For this channel model, the cut-set bound is sensitive to the cooperation noises, but not sensitive enough. For all nonzero values of cooperation noise variances, our outer bound strictly improves upon the cut-set outer bound. Moreover, as the cooperation noises become large, our outer bound collapses to the capacity region of the Gaussian MAC without cooperation.

Thirdly, we investigate a Gaussian IC with user-cooperation (ICUC). For this channel model, the cut-set bound is again sensitive to cooperation noise variances as in the case of MAC-UC channel model, but not sensitive enough. We demonstrate that our outer bound strictly improves upon the cut-set bound for all nonzero values of cooperation noise variances.
\end{abstract}

Index Terms-Dependence balance, generalized feedback, user cooperation, noisy feedback.

\section{INTRODUCTION}

I $\mathrm{T}$ is well known that noiseless feedback can increase the capacity region of the discrete memoryless multiple access channel as was shown by Gaarder and Wolf in [1]. The multiple

Manuscript received December 10, 2008; revised August 16, 2010; accepted December 21, 2010. Date of current version June 22, 2011. This work was supported by NSF Grants CCF 04-47613, CCF 05-14846, CNS 07-16311, and CCF 07-29127, and was presented in part at the IEEE International Conference on Communications, Dresden, Germany, June 2009, the IEEE Information Theory Workshop, Volos, Greece, June 2009, and the IEEE International Symposium on Information Theory, Seoul, South Korea, June 2009.

R. Tandon was with the Department of Electrical and Computer Engineering, University of Maryland, College Park, MD 20742 USA. He is now with the Department of Electrical Engineering, Princeton University, Princeton, NJ 08544 USA (e-mail: rtandon@ princeton.edu).

S. Ulukus is with the Department of Electrical and Computer Engineering, University of Maryland, College Park, MD 20742 USA (e-mail: ulukus@umd. edu).

Communicated by A. J. Goldsmith, Associate Editor for Communications.

Digital Object Identifier 10.1109/TIT.2011.2145150 access channel with generalized feedback (MAC-GF) was first introduced by Carleial [2]. The model therein allows for different feedback signals at the two transmitters. For this channel model, Carleial [2] obtained an achievable rate region using block Markov superposition encoding and windowed decoding. An improvement over this achievable rate region was obtained by Willems et al. in [3] by using block Markov superposition encoding combined with backwards decoding.

Inspired from the uplink MAC-GF channel model, the interference channel with generalized feedback (IC-GF) was studied in [4], [5], (also see the references therein) where achievable rate regions were obtained. It was shown in [4] and [5] that for the Gaussian interference channel with user cooperation (IC-UC), the overheard information at the transmitters has a dual effect of enabling cooperation and mitigating interference, thereby providing improved achievable rates compared to the best known evaluation of the Han-Kobayashi achievable rate region [6], [7].

As far as the converses are concerned for the MAC-GF and the IC-GF, a well known outer bound is the cut-set outer bound [8, Th. 14.10.1]. The cut-set bound allows all input distributions, thereby permitting arbitrary correlation between the channel inputs and hence is seemingly loose. The idea of dependence balance was first introduced by Hekstra and Willems [9] to obtain outer bounds on the capacity region of single output two-way channel. In contrast to the cut-set bound, the dependence balance bound provides an additional nontrivial restriction over the set of allowable input distributions thus leading to a potentially tighter outer bound. In the same paper [9], the authors give a variant of this bound for the two-user discrete memoryless MAC with noiseless feedback from the receiver.

In this paper, we use the idea of dependence balance to obtain new outer bounds on the capacity regions of the MAC-GF and the IC-GF. To show the usefulness of our outer bounds, we will consider three different channel models.

We first consider the Gaussian MAC with different noisy feedback signals at the two transmitters. Specifically, transmitter $k, k=1,2$, receives a feedback $Y_{F_{k}}=Y+Z_{k}$, where $Y$ is the received signal and $Z_{k}$ is zero-mean, Gaussian random variable with variance $\sigma_{Z_{k}}^{2}$. The capacity region is only known when feedback is noiseless, i.e., $Y_{F_{1}}=Y_{F_{2}}=Y$, in which case the feedback capacity region equals the cut-set outer bound, as was shown by Ozarow [10]. For the case of noisy feedback in consideration, the cut-set outer bound is insensitive to the noise in feedback links, i.e., it is not sensitive to the variances of $Z_{1}$ and $Z_{2}$. We show that our outer bound strictly improves upon the cut-set bound for all nonzero values of $\left(\sigma_{Z_{1}}^{2}, \sigma_{Z_{2}}^{2}\right)$. Furthermore, as $\left(\sigma_{Z_{1}}^{2}, \sigma_{Z_{2}}^{2}\right)$ become large, our outer bound collapses to the capacity region of the Gaussian MAC without feedback. 


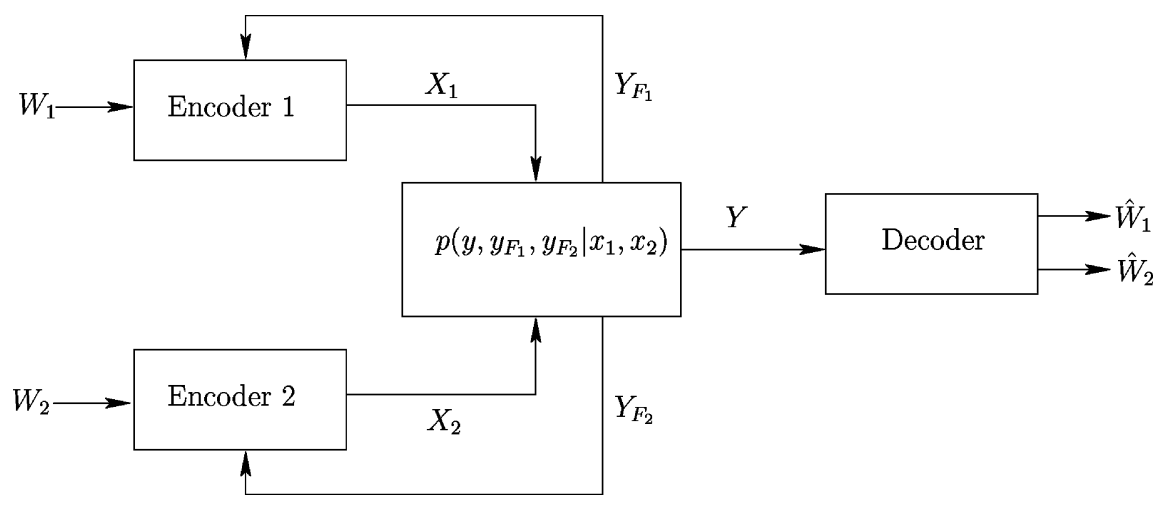

Fig. 1. Multiple access channel with generalized feedback (MAC-GF).

We remark here that the renewed interest in dependence balance bounds can be attributed to [11], where dependence balance bounds were used to obtain capacity results for a class of discrete memoryless networks. In [12], novel dependence balance bounds were obtained for the $K$-user Gaussian MAC with noiseless feedback. Furthermore, applying the idea of dependence balance to obtain improved outer bounds for Gaussian MAC with noisy feedback was proposed by Gastpar and Kramer in [13].

Secondly, we investigate the Gaussian MAC with transmitter cooperation. Sendonaris, Erkip, and Aazhang [14] studied a model where each transmitter receives a version of the other transmitter's current channel input corrupted with additive white Gaussian noise. They named this model as the user cooperation model. This model is particularly suitable for a wireless setting since the transmitters can potentially overhear each other. An achievable rate region for the user cooperation model was given in [14] using the result of [3] and was shown to strictly exceed the rate region if the transmitters ignore the overheard signals.

We evaluate our outer bound for the user cooperation setting described above. In contrast to the case of noisy feedback, the cut-set bound for the user cooperation model is sensitive to cooperation noise variances, but not too sensitive. Intuitively speaking, as the backward noise variances become large, one would expect the cut-set bound to collapse to the capacity region of the MAC without feedback. Instead, the cut-set bound converges to the capacity region of the Gaussian MAC with noiseless output feedback [10]. On the other hand, in the limit when cooperation noise variances become too large, our bound converges to the capacity region of the Gaussian MAC with no cooperation. For all nonzero and finite values of cooperation noise variances, our outer bound strictly improves upon the cut-set outer bound. Our dependence balance based outer bound coincides with the cut-set bound only when the backward noise variance is identically zero and both outer bounds collapse to the total cooperation line.

Thirdly, we evaluate our outer bound for the Gaussian IC with user cooperation (IC-UC). For all nonzero and finite values of cooperation noise variances, our outer bound strictly improves upon the cut-set outer bound. We remark here that the approach of dependence balance was also used in [15] to obtain an improved sum-rate upper bound for the Gaussian IC with common, noisy feedback from the receivers.
Evaluation of our outer bounds for MAC-NF, MAC-UC and IC-UC is not straightforward since our outer bounds are expressed in terms of a union of probability densities of three random variables, one of which is an auxiliary random variable. Moreover, these unions are over all such densities which satisfy a nontrivial dependence balance constraint. We overcome this difficulty by proving separately for all three models in consideration, that it is sufficient to consider jointly Gaussian input distributions, satisfying the dependence balance constraint, when evaluating our outer bounds. The proof methodology for showing this claim is entirely different for each of the cases of noisy feedback and user cooperation models. In particular, for the case of MAC-NF, we make use of a recently discovered multivariate generalization [16] of Costa's entropy power inequality (EPI) [17] along with some properties of $3 \times 3$ covariance matrices to obtain this result. On the other hand, for the case of MAC-UC and IC-UC, we do not need EPI to show this result and our proof closely follows the proof of a recent result by Bross, Lapidoth, and Wigger [18], [19] for the Gaussian MAC with conferencing encoders. This approach has also been recently used for other channel models in [20], [21]. The structure of dependence balance constraints for the channel models in consideration are of different form, which explains the different methodology of proofs. We believe that the proof methodology developed for evaluating our outer bounds could be helpful for other multiuser information theoretic problems.

\section{SYSTEM MODEL}

\section{A. MAC With Generalized Feedback}

A discrete memoryless two-user multiple access channel with generalized feedback (MAC-GF) (see Fig. 1) is defined by two input alphabets $\mathcal{X}_{1}$ and $\mathcal{X}_{2}$, an output alphabet for the receiver $\mathcal{Y}$, feedback output alphabets $\mathcal{Y}_{F_{1}}$ and $\mathcal{Y}_{F_{2}}$ at transmitters 1 and 2, respectively, and a probability transition function $p\left(y, y_{F_{1}}, y_{F_{2}} \mid x_{1}, x_{2}\right)$, defined for all triples $\left(y, y_{F_{1}}, y_{F_{2}}\right) \in \mathcal{Y} \times \mathcal{Y}_{F_{1}} \times \mathcal{Y}_{F_{2}}$, for every pair $\left(x_{1}, x_{2}\right) \in \mathcal{X}_{1} \times \mathcal{X}_{2}$.

A $\left(n, M_{1}, M_{2}, P_{e}\right)$ code for the MAC-GF consists of two sets of encoding functions $f_{1 i}: \mathcal{M}_{1} \times \mathcal{Y}_{F_{1}}^{i-1} \rightarrow \mathcal{X}_{1}, f_{2 i}: \mathcal{M}_{2} \times$ $\mathcal{Y}_{F_{2}}^{i-1} \rightarrow \mathcal{X}_{2}$ for $i=1, \ldots, n$ and a decoding function $g$ : $\mathcal{Y}^{n^{2}} \rightarrow \mathcal{M}_{1} \times \mathcal{M}_{2}$. The two transmitters produce independent and uniformly distributed messages $W_{1} \in\left\{1, \ldots, M_{1}\right\}$ and $W_{2} \in\left\{1, \ldots, M_{2}\right\}$, respectively, and transmit them through $n$ 


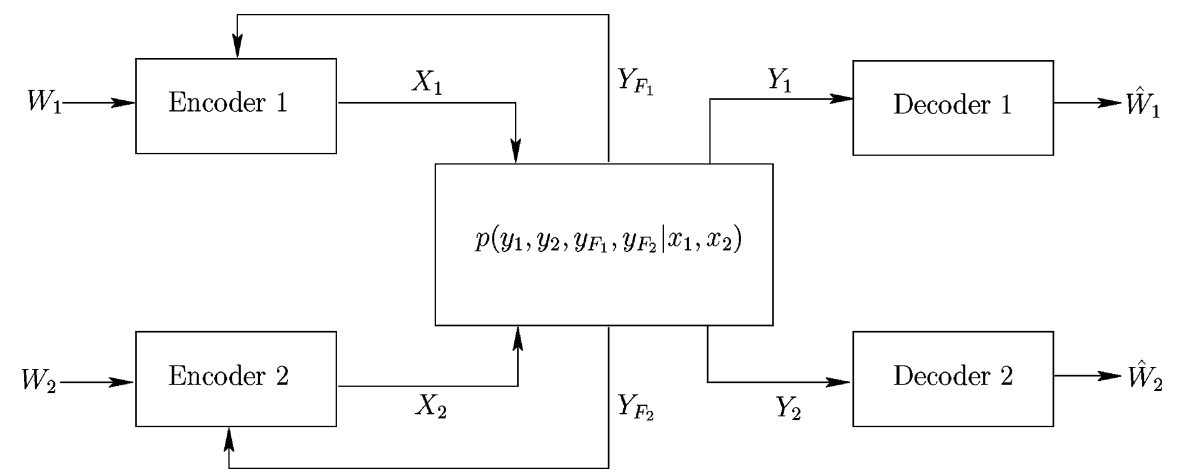

Fig. 2. Interference channel with generalized feedback (IC-GF).

channel uses. The average error probability is defined as, $P_{e}=$ $\operatorname{Pr}\left[\left(\hat{W}_{1}, \hat{W}_{2}\right) \neq\left(W_{1}, W_{2}\right)\right]$. A rate pair $\left(R_{1}, R_{2}\right)$ is said to be achievable for MAC-GF if for any $\epsilon \geq 0$, there exists a pair of $n$ encoding functions $\left\{f_{1 i}\right\}_{i=1}^{n},\left\{f_{2 i}\right\}_{i=1}^{n}$, and a decoding function $g: \mathcal{Y}^{n} \rightarrow \mathcal{M}_{1} \times \mathcal{M}_{2}$ such that $R_{1} \leq \log \left(M_{1}\right) / n, R_{2} \leq$ $\log \left(M_{2}\right) / n$ and $P_{e} \leq \epsilon$ for sufficiently large $n$. The capacity region of MAC-GF is the closure of the set of all achievable rate pairs $\left(R_{1}, R_{2}\right)$.

\section{B. IC With Generalized Feedback}

A discrete memoryless two-user interference channel with generalized feedback (IC-GF) (see Fig. 2) is defined by two input alphabets $\mathcal{X}_{1}$ and $\mathcal{X}_{2}$, two output alphabets $\mathcal{Y}_{1}$ and $\mathcal{Y}_{2}$ at receivers 1 and 2, respectively, two feedback output alphabets $\mathcal{Y}_{F_{1}}$ and $\mathcal{Y}_{F_{2}}$ at transmitters 1 and 2, respectively, and a probability transition function $p\left(y_{1}, y_{2}, y_{F_{1}}, y_{F_{2}} \mid x_{1}, x_{2}\right)$, defined for all quadruples $\left(y_{1}, y_{2}, y_{F_{1}}, y_{F_{2}}\right) \in \mathcal{Y}_{1} \times \mathcal{Y}_{2} \times \mathcal{Y}_{F_{1}} \times \mathcal{Y}_{F_{2}}$, for every pair $\left(x_{1}, x_{2}\right) \in \mathcal{X}_{1} \times \mathcal{X}_{2}$.

A $\left(n, M_{1}, M_{2}, P_{e}^{(1)}, P_{e}^{(2)}\right)$ code for IC-GF consists of two sets of encoding functions $f_{1 i}: \mathcal{M}_{1} \times \mathcal{Y}_{F_{1}}^{i-1} \rightarrow \mathcal{X}_{1}, f_{2 i}$ : $\mathcal{M}_{2} \times \mathcal{Y}_{F_{2}}^{i-1} \rightarrow \mathcal{X}_{2}$ for $i=1, \ldots, n$ and two decoding functions $g_{1}: \mathcal{Y}_{1}^{n} \rightarrow \mathcal{M}_{1}$ and $g_{2}: \mathcal{Y}_{2}^{n} \rightarrow \mathcal{M}_{2}$. The two transmitters produce independent and uniformly distributed messages $W_{1} \in$ $\left\{1, \ldots, M_{1}\right\}$ and $W_{2} \in\left\{1, \ldots, M_{2}\right\}$, respectively, and transmit them through $n$ channel uses. The average error probability at receivers 1 and 2 are defined as, $P_{e}^{(k)}=\operatorname{Pr}\left[\hat{W}_{k} \neq W_{k}\right]$ for $k=1,2$. A rate pair $\left(R_{1}, R_{2}\right)$ is said to be achievable for IC-GF if for any pair $\epsilon_{1} \geq 0, \epsilon_{2} \geq 0$, there exists a pair of $n$ encoding functions $\left\{f_{1 i}\right\}_{i=1}^{n},\left\{f_{2 i}\right\}_{i=1}^{n}$, and a pair of decoding functions $\left(g_{1}, g_{2}\right)$ such that $R_{1} \leq \log \left(M_{1}\right) / n, R_{2} \leq \log \left(M_{2}\right) / n$ and $P_{e}^{(k)} \leq \epsilon_{k}$ for sufficiently large $n$, for $k=1,2$. The capacity region of IC-GF is the closure of the set of all achievable rate pairs $\left(R_{1}, R_{2}\right)$.

\section{Cut-Set Outer BoundS}

A general outer bound on the capacity region of a multiterminal network is the cut-set outer bound [8, Th. 14.10.1]. The cut-set outer bound for MAC-GF is given by

$$
\begin{aligned}
\mathcal{C S}^{M A C}=\left\{\left(R_{1}, R_{2}\right):\right. & R_{1} \leq I\left(X_{1} ; Y, Y_{F_{2}} \mid X_{2}\right) \\
& R_{2} \leq I\left(X_{2} ; Y, Y_{F_{1}} \mid X_{1}\right) \\
& \left.R_{1}+R_{2} \leq I\left(X_{1}, X_{2} ; Y\right)\right\}
\end{aligned}
$$

where the random variables $X_{1}, X_{2}$ and $\left(Y, Y_{F_{1}}, Y_{F_{2}}\right)$ have the joint distribution

$$
p\left(x_{1}, x_{2}, y, y_{F_{1}}, y_{F_{2}}\right)=p\left(x_{1}, x_{2}\right) p\left(y, y_{F_{1}}, y_{F_{2}} \mid x_{1}, x_{2}\right) .
$$

The cut-set outer bound for IC-GF is given by

$$
\begin{aligned}
\mathcal{C S}^{I C}=\left\{\left(R_{1}, R_{2}\right):\right. & R_{1} \leq I\left(X_{1}, X_{2} ; Y_{1}\right) \\
& R_{2} \leq I\left(X_{1}, X_{2} ; Y_{2}\right) \\
& R_{1} \leq I\left(X_{1} ; Y_{1}, Y_{F_{2}} \mid X_{2}\right) \\
& R_{2} \leq I\left(X_{2} ; Y_{2}, Y_{F_{1}} \mid X_{1}\right) \\
& \left.R_{1}+R_{2} \leq I\left(X_{1}, X_{2} ; Y_{1}, Y_{2}\right)\right\}
\end{aligned}
$$

where the random variables $X_{1}, X_{2}$ and $\left(Y_{1}, Y_{2}, Y_{F_{1}}, Y_{F_{2}}\right)$ have the joint distribution

$$
\begin{aligned}
& p\left(x_{1}, x_{2}, y_{1}, y_{2}, y_{F_{1}}, y_{F_{2}}\right) \\
& =p\left(x_{1}, x_{2}\right) p\left(y_{1}, y_{2}, y_{F_{1}}, y_{F_{2}} \mid x_{1}, x_{2}\right) .
\end{aligned}
$$

We remark here that the outer bounds $\mathcal{C S}^{M A C}$ and $\mathcal{C S}^{I C}$ stated above are obtained by taking a union over all input distributions of the forms (4) and (10), respectively.

The cut-set bound is seemingly loose since it allows arbitrary correlation among channel inputs by permitting arbitrary input distributions $p\left(x_{1}, x_{2}\right)$. Using the approach of dependence balance, we will obtain outer bounds for MAC-GF and IC-GF which restrict the corresponding set of input distributions for both channel models. In particular, our outer bounds only permit those input distributions which satisfy the respective nontrivial dependence balance constraints.

\section{A NEW OUTER BOUND FOR MAC-GF}

Theorem 1: The capacity region of MAC-GF is contained in the region

$$
\begin{aligned}
\mathcal{D B}^{M A C} & =\left\{\left(R_{1}, R_{2}\right):\right. \\
& R_{1} \leq I\left(X_{1} ; Y, Y_{F_{1}}, Y_{F_{2}} \mid X_{2}, T\right) \\
& R_{2} \leq I\left(X_{2} ; Y, Y_{F_{1}}, Y_{F_{2}} \mid X_{1}, T\right) \\
& R_{1}+R_{2} \leq I\left(X_{1}, X_{2} ; Y, Y_{F_{1}}, Y_{F_{2}} \mid T\right) \\
& \left.R_{1}+R_{2} \leq I\left(X_{1}, X_{2} ; Y\right)\right\}
\end{aligned}
$$




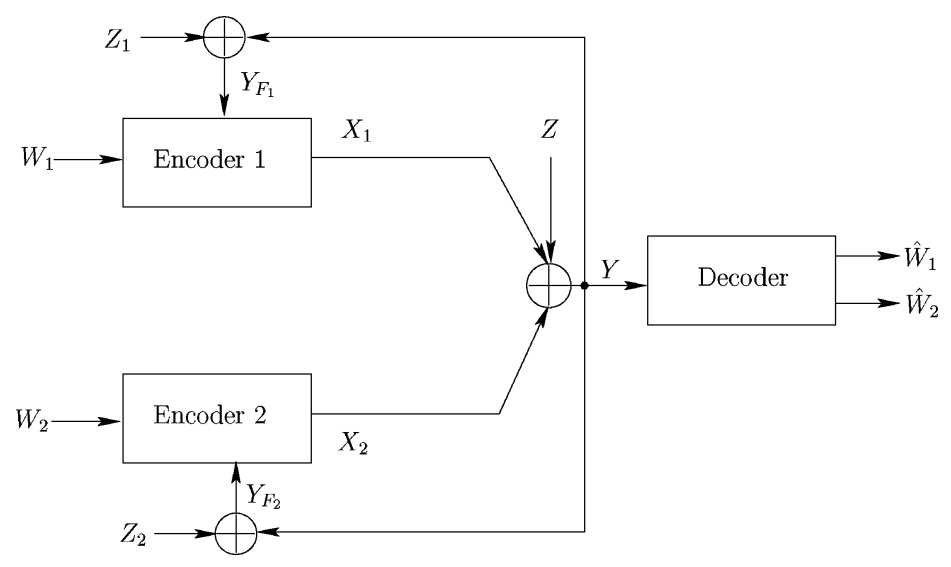

Fig. 3. Gaussian MAC with noisy feedback.

where the random variables $\left(T, X_{1}, X_{2}, Y, Y_{F_{1}}, Y_{F_{2}}\right)$ have the joint distribution

$$
p\left(t, x_{1}, x_{2}, y, y_{F_{1}}, y_{F_{2}}\right)=p\left(t, x_{1}, x_{2}\right) p\left(y, y_{F_{1}}, y_{F_{2}} \mid x_{1}, x_{2}\right)
$$

and also satisfy the following dependence balance bound

$$
I\left(X_{1} ; X_{2} \mid T\right) \leq I\left(X_{1} ; X_{2} \mid Y_{F_{1}}, Y_{F_{2}}, T\right)
$$

where the random variable $T$ is subject to a cardinality constraint $|\mathcal{T}| \leq\left|\mathcal{X}_{1}\right|\left|\mathcal{X}_{2}\right|+3$.

The Proof of Theorem 1 is given in the Appendix.

\section{A NEW OUTER BOUND FOR IC-GF}

Theorem 2: The capacity region of IC-GF is contained in the region

$$
\begin{aligned}
\mathcal{D B}^{I C} & =\left\{\left(R_{1}, R_{2}\right):\right. \\
& R_{1} \leq I\left(X_{1}, X_{2} ; Y_{1}\right) \\
& R_{2} \leq I\left(X_{1}, X_{2} ; Y_{2}\right) \\
& R_{1} \leq I\left(X_{1} ; Y_{1}, Y_{F_{1}}, Y_{F_{2}} \mid X_{2}, T\right) \\
& R_{2} \leq I\left(X_{2} ; Y_{2}, Y_{F_{1}}, Y_{F_{2}} \mid X_{1}, T\right) \\
& R_{1}+R_{2} \leq I\left(X_{1}, X_{2} ; Y_{1}, Y_{2}, Y_{F_{1}}, Y_{F_{2}} \mid T\right) \\
& \left.R_{1}+R_{2} \leq I\left(X_{1}, X_{2} ; Y_{1}, Y_{2}\right)\right\}
\end{aligned}
$$

where the random variables $\left(T, X_{1}, X_{2}, Y_{1}, Y_{2}, Y_{F_{1}}, Y_{F_{2}}\right)$ have the joint distribution

$$
\begin{aligned}
p\left(t, x_{1}, x_{2}, y_{1}, y_{2}, y_{F_{1}}, y_{F_{2}}\right) & \\
& =p\left(t, x_{1}, x_{2}\right) p\left(y_{1}, y_{2}, y_{F_{1}}, y_{F_{2}} \mid x_{1}, x_{2}\right)
\end{aligned}
$$

and also satisfy the following dependence balance bound

$$
I\left(X_{1} ; X_{2} \mid T\right) \leq I\left(X_{1} ; X_{2} \mid Y_{F_{1}}, Y_{F_{2}}, T\right)
$$

where the random variable $T$ is subject to a cardinality constraint $|\mathcal{T}| \leq\left|\mathcal{X}_{1}\right|\left|\mathcal{X}_{2}\right|+3$.

The Proof of Theorem 2 is given in the Appendix.

We remark that Theorems 1 and 2 can be strengthened by introducing two auxiliary random variables $T_{1}$ and $T_{2}$. We also note here that one can obtain fixed and adaptive parallel channel extensions of the dependence balance based bounds in a similar fashion as in [9]. The parallel channel extensions could potentially improve upon the outer bounds derived in this paper. For the scope of this paper, we will only use Theorems 1 and 2. In the next three sections, we will consider specific channel models of MAC with noisy feedback, MAC with user cooperation, and IC with user cooperation.

We also remark here that dependence balance approach was first applied by Gastpar and Kramer for the Gaussian MAC with noisy feedback in [13] and for the Gaussian IC with noisy feedback (IC-NF) in [15]. An interesting Lagrangian based approach was proposed in [15] to partially evaluate the dependence balance based outer bound for the Gaussian IC-NF and it was shown that dependence balance based bounds strictly improve upon the cut-set outer bound. For this reason, we do not consider the Gaussian IC-NF in this paper.

\section{Gaussian MAC With NoIsy FeEdBack}

We first consider the Gaussian MAC with noisy feedback (see Fig. 3). The channel model is given as,

$$
\begin{aligned}
Y & =X_{1}+X_{2}+Z \\
Y_{F_{1}} & =Y+Z_{1} \\
Y_{F_{2}} & =Y+Z_{2}
\end{aligned}
$$

where $Z, Z_{1}$ and $Z_{2}$ are independent, zero-mean, Gaussian random variables with variances $\sigma_{Z}^{2}, \sigma_{Z_{1}}^{2}$ and $\sigma_{Z_{2}}^{2}$, respectively. Moreover, the channel inputs are subject to average power constraints, $E\left[X_{1}^{2}\right] \leq P_{1}$ and $E\left[X_{2}^{2}\right] \leq P_{2}$. Note that the channel model described above has a special probability structure, namely

$$
p\left(y, y_{F_{1}}, y_{F_{2}} \mid x_{1}, x_{2}\right)=p\left(y \mid x_{1}, x_{2}\right) p\left(y_{F_{1}} \mid y\right) p\left(y_{F_{2}} \mid y\right)
$$

For any MAC-GF with a transition probability in the form of (28), we have the following specialized version of Theorem 1.

Theorem 3: The capacity region of any MAC-GF, with a transition probability in the form of (28), is contained in the region

$$
\begin{aligned}
\mathcal{D B}_{N F}^{M A C}=\left\{\left(R_{1}, R_{2}\right):\right. & R_{1} \leq I\left(X_{1} ; Y \mid X_{2}, T\right) \\
& R_{2} \leq I\left(X_{2} ; Y \mid X_{1}, T\right) \\
& \left.R_{1}+R_{2} \leq I\left(X_{1}, X_{2} ; Y \mid T\right)\right\}
\end{aligned}
$$




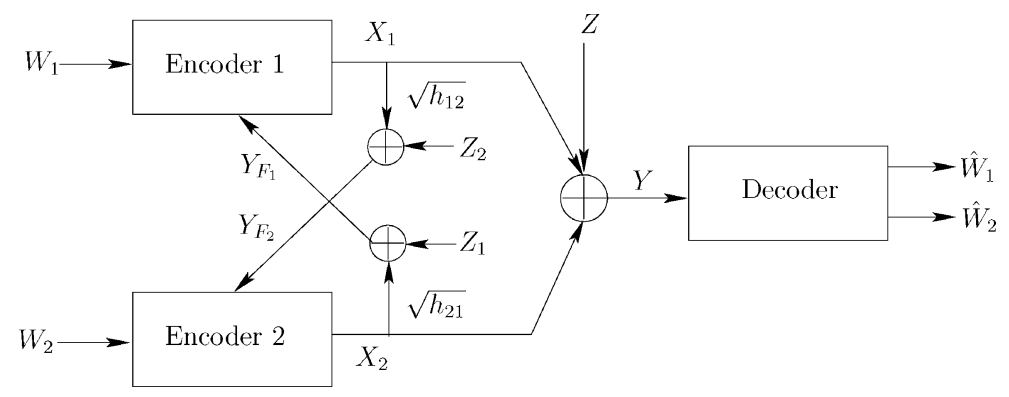

Fig. 4. Gaussian MAC with user cooperation.

where the random variables $\left(T, X_{1}, X_{2}, Y, Y_{F_{1}}, Y_{F_{2}}\right)$ have the joint distribution

$$
\begin{aligned}
& p\left(t, x_{1}, x_{2}, y, y_{F_{1}}, y_{F_{2}}\right) \\
& \quad=p\left(t, x_{1}, x_{2}\right) p\left(y \mid x_{1}, x_{2}\right) p\left(y_{F_{1}} \mid y\right) p\left(y_{F_{2}} \mid y\right)
\end{aligned}
$$

and also satisfy the following dependence balance bound

$$
I\left(X_{1} ; X_{2} \mid T\right) \leq I\left(X_{1} ; X_{2} \mid Y_{F_{1}}, Y_{F_{2}}, T\right)
$$

where the random variable $T$ is subject to a cardinality constraint $|\mathcal{T}| \leq\left|\mathcal{X}_{1}\right|\left|\mathcal{X}_{2}\right|+3$.

The Proof of Theorem 3 follows from Theorem 1 by using (28).

In Section X, we will show that it suffices to consider jointly Gaussian ( $\left.T, X_{1}, X_{2}\right)$ satisfying (33) when evaluating Theorem 3 for the Gaussian MAC with noisy feedback described in (25)-(27).

\section{GAUSSIAN MAC With USER COOPERATION}

In this section, we consider the Gaussian MAC with user cooperation [14], where each transmitter receives a noisy version of the other transmitter's channel input. The user cooperation model (see Fig. 4) is a special instance of a MAC-GF, where the channel outputs are described as

$$
\begin{aligned}
Y & =\sqrt{h_{10}} X_{1}+\sqrt{h_{20}} X_{2}+Z \\
Y_{F_{1}} & =\sqrt{h_{21}} X_{2}+Z_{1} \\
Y_{F_{2}} & =\sqrt{h_{12}} X_{1}+Z_{2}
\end{aligned}
$$

where $Z, Z_{1}$ and $Z_{2}$ are independent, zero-mean, Gaussian random variables with variances $\sigma_{Z}^{2}, \sigma_{Z_{1}}^{2}$ and $\sigma_{Z_{2}}^{2}$, respectively. The channel gains $h_{10}, h_{20}, h_{12}$ and $h_{21}$ are assumed to be fixed and known at all terminals. Moreover, the channel inputs are subject to average power constraints, $E\left[X_{1}^{2}\right] \leq P_{1}$ and $E\left[X_{2}^{2}\right] \leq P_{2}$. Note that the channel model described above has a special probability structure, namely

$$
p\left(y, y_{F_{1}}, y_{F_{2}} \mid x_{1}, x_{2}\right)=p\left(y \mid x_{1}, x_{2}\right) p\left(y_{F_{1}} \mid x_{2}\right) p\left(y_{F_{2}} \mid x_{1}\right)
$$

For any MAC-GF with a transition probability in the form of (37), we have the following specialized version of Theorem 1.
Theorem 4: The capacity region of any MAC-GF with a transition probability in the form of (37), is contained in the region

$$
\begin{aligned}
\mathcal{D} \mathcal{B}_{U C}^{M A C}=\{ & \left(R_{1}, R_{2}\right): \\
& R_{1} \leq I\left(X_{1} ; Y, Y_{F_{2}} \mid X_{2}, T\right) \\
& R_{2} \leq I\left(X_{2} ; Y, Y_{F_{1}} \mid X_{1}, T\right) \\
& R_{1}+R_{2} \leq I\left(X_{1}, X_{2} ; Y, Y_{F_{1}}, Y_{F_{2}} \mid T\right) \\
& \left.R_{1}+R_{2} \leq I\left(X_{1}, X_{2} ; Y\right)\right\}
\end{aligned}
$$

where the random variables $\left(T, X_{1}, X_{2}, Y, Y_{F_{1}}, Y_{F_{2}}\right)$ have the joint distribution

$$
\begin{aligned}
& p\left(t, x_{1}, x_{2}, y, y_{F_{1}}, y_{F_{2}}\right) \\
& \quad=p\left(t, x_{1}, x_{2}\right) p\left(y \mid x_{1}, x_{2}\right) p\left(y_{F_{1}} \mid x_{2}\right) p\left(y_{F_{2}} \mid x_{1}\right)
\end{aligned}
$$

and also satisfy the following dependence balance bound

$$
I\left(X_{1} ; X_{2} \mid T\right) \leq I\left(X_{1} ; X_{2} \mid Y_{F_{1}}, Y_{F_{2}}, T\right)
$$

where the random variable $T$ is subject to a cardinality constraint $|\mathcal{T}| \leq\left|\mathcal{X}_{1}\right|\left|\mathcal{X}_{2}\right|+3$

The Proof of Theorem 4 follows from Theorem 1 by using (37).

In Section XI, we will show that it suffices to consider jointly Gaussian ( $\left.T, X_{1}, X_{2}\right)$ satisfying (43) when evaluating Theorem 4 for the Gaussian MAC with user cooperation described in (34)-(36).

\section{GAUSSIAN IC WITH USER COOPERATION}

In this section, we will evaluate our outer bound for an IC with user cooperation [4], [5], where the transmitters receive noisy versions of the other transmitter's channel input. The user cooperation model (see Fig. 5) is a special instance of an IC-GF, where the channel outputs are described as

$$
\begin{aligned}
Y_{1} & =X_{1}+\sqrt{b} X_{2}+N_{1} \\
Y_{2} & =\sqrt{a} X_{1}+X_{2}+N_{2} \\
Y_{F_{1}} & =\sqrt{h_{21}} X_{2}+Z_{1} \\
Y_{F_{2}} & =\sqrt{h_{12}} X_{1}+Z_{2}
\end{aligned}
$$

where $N_{1}, N_{2}, Z_{1}$ and $Z_{2}$ are independent, zero-mean, Gaussian random variables with variances $\sigma_{N_{1}}^{2}, \sigma_{N_{2}}^{2}, \sigma_{Z_{1}}^{2}$ and $\sigma_{Z_{2}}^{2}$, respectively. The channel gains $a, b, h_{12}$ and $h_{21}$ are assumed to be fixed and known at all terminals. Moreover, the channel inputs are subject to average power constraints 


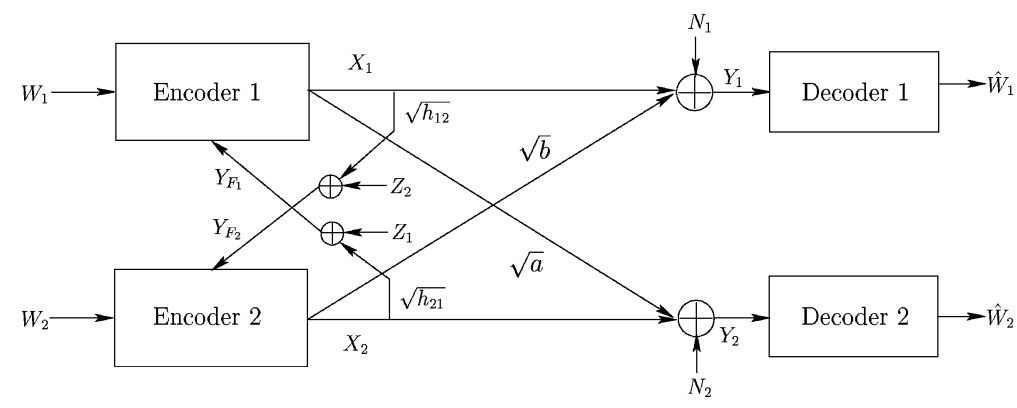

Fig. 5. Gaussian IC with user cooperation.

$E\left[X_{1}^{2}\right] \leq P_{1}$ and $E\left[X_{2}^{2}\right] \leq P_{2}$. Note that the channel model described above has a special probability structure, namely

$$
\begin{aligned}
p\left(y_{1}, y_{2}, y_{F_{1}}, y_{F_{2}} \mid x_{1}, x_{2}\right) & \\
= & p\left(y_{1}, y_{2} \mid x_{1}, x_{2}\right) p\left(y_{F_{1}} \mid x_{2}\right) p\left(y_{F_{2}} \mid x_{1}\right) .
\end{aligned}
$$

For any IC-GF with a transition probability in the form of (48), we have the following specialized version of Theorem 2 .

Theorem 5: The capacity region of any IC-GF with a transition probability in the form of (48), is contained in the region

$$
\begin{aligned}
\mathcal{D B} \mathcal{B}_{U C}^{I C}=\{ & \left(R_{1}, R_{2}\right): \\
& R_{1} \leq I\left(X_{1}, X_{2} ; Y_{1}\right) \\
& R_{2} \leq I\left(X_{1}, X_{2} ; Y_{2}\right) \\
& R_{1} \leq I\left(X_{1} ; Y_{1}, Y_{F_{2}} \mid X_{2}, T\right) \\
& R_{2} \leq I\left(X_{2} ; Y_{2}, Y_{F_{1}} \mid X_{1}, T\right) \\
& R_{1}+R_{2} \leq I\left(X_{1}, X_{2} ; Y_{1}, Y_{2}, Y_{F_{1}}, Y_{F_{2}} \mid T\right) \\
& \left.R_{1}+R_{2} \leq I\left(X_{1}, X_{2} ; Y_{1}, Y_{2}\right)\right\}
\end{aligned}
$$

where the random variables $\left(T, X_{1}, X_{2}, Y_{1}, Y_{2}, Y_{F_{1}}, Y_{F_{2}}\right)$ have the joint distribution

$$
\begin{aligned}
& p\left(t, x_{1}, x_{2}, y_{1}, y_{2}, y_{F_{1}}, y_{F_{2}}\right) \\
& \quad=p\left(t, x_{1}, x_{2}\right) p\left(y_{1}, y_{2} \mid x_{1}, x_{2}\right) p\left(y_{F_{1}} \mid x_{2}\right) p\left(y_{F_{2}} \mid x_{1}\right)
\end{aligned}
$$

and also satisfy the following dependence balance bound

$$
I\left(X_{1} ; X_{2} \mid T\right) \leq I\left(X_{1} ; X_{2} \mid Y_{F_{1}}, Y_{F_{2}}, T\right)
$$

where the random variable $T$ is subject to a cardinality constraint $|\mathcal{T}| \leq\left|\mathcal{X}_{1}\right|\left|\mathcal{X}_{2}\right|+3$.

The Proof of Theorem 5 follows from Theorem 2 by using (48).

In Section XII, we will show that it suffices to consider jointly Gaussian $\left(T, X_{1}, X_{2}\right)$ satisfying (56) when evaluating Theorem 5 for the Gaussian IC with user cooperation described in (44)-(47).

\section{OUtLINE FOR EVALUATING $\mathcal{D} \mathcal{B}_{N F}^{M A C}$, $\mathcal{D} \mathcal{B}_{U C}^{M A C}$, AND $\mathcal{D} \mathcal{B}_{U C}^{I C}$}

In this section, we outline the common approach for evaluation of our outer bounds, $\mathcal{D B} \mathcal{B}_{N F}^{M A C}$ for the Gaussian MAC with noisy feedback, $\mathcal{D} \mathcal{B}_{U C}^{M A C}$ for the Gaussian MAC with user-cooperation and $\mathcal{D} \mathcal{B}_{U C}^{I C}$ for the Gaussian IC with user-coopera-

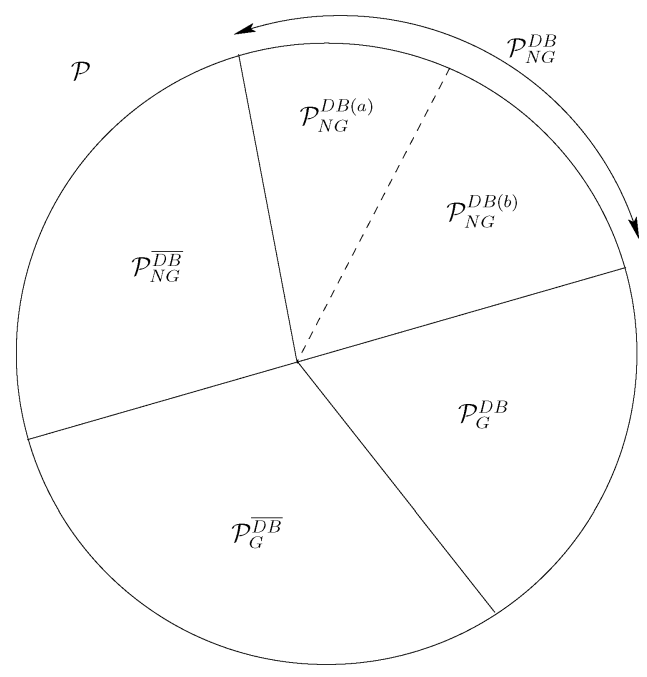

Fig. 6. Partition of the set of input distributions $\mathcal{P}$.

tion. The main difficulty in evaluating these bounds is to identify the optimal selection of joint densities of $\left(T, X_{1}, X_{2}\right)$. Our aim will be to prove that it is sufficient to consider jointly Gaussian ( $T, X_{1}, X_{2}$ ) satisfying (33) for MAC with noisy feedback, (43) for MAC with user cooperation, and (56) for IC with user cooperation, respectively, when evaluating the corresponding outer bounds.

First note that the three outer bounds, namely $\mathcal{D} \mathcal{B}_{N F}^{M A C}$, $\mathcal{D} \mathcal{B}_{U C}^{M A C}$ and $\mathcal{D} \mathcal{B}_{U C}^{I C}$ have a similar structure, i.e., all outer bounds involve taking a union over joint densities of $\left(T, X_{1}, X_{2}\right)$ satisfying the constraints (33), (43), and (56), respectively. Let us symbolically denote these constraints as a variable $(D B)$, where $(D B)=(33)$ for MAC with noisy feedback, $(D B)=(43)$ for MAC with user cooperation, and $(D B)=(56)$ for IC with user cooperation.

We begin by considering the set of all distributions of three random variables $\left(T, X_{1}, X_{2}\right)$ which satisfy the power constraints, $E\left[X_{1}^{2}\right] \leq P_{1}$ and $E\left[X_{2}^{2}\right] \leq P_{2}$. Let us formally define this set of input distributions as

$$
\mathcal{P}=\left\{p\left(t, x_{1}, x_{2}\right): E\left[X_{1}^{2}\right] \leq P_{1}, E\left[X_{2}^{2}\right] \leq P_{2}\right\} .
$$

For simplicity, we abbreviate jointly Gaussian distributions as $\mathcal{J G}$ and distributions which are not jointly Gaussian as $\mathcal{N G}$. We first partition $\mathcal{P}$ into two disjoint subsets

$$
\begin{aligned}
\mathcal{P}_{G} & =\left\{p\left(t, x_{1}, x_{2}\right) \in \mathcal{P}:\left(T, X_{1}, X_{2}\right) \text { are } \mathcal{J G}\right\} \\
\mathcal{P}_{N G} & =\left\{p\left(t, x_{1}, x_{2}\right) \in \mathcal{P}:\left(T, X_{1}, X_{2}\right) \text { are } \mathcal{N G}\right\}
\end{aligned}
$$


We further individually partition the sets $\mathcal{P}_{G}$ and $\mathcal{P}_{N G}$, respectively, as

$$
\begin{aligned}
\mathcal{P}_{G}^{D B}= & \left\{p\left(t, x_{1}, x_{2}\right) \in \mathcal{P}_{G}:\left(T, X_{1}, X_{2}\right) \text { satisfy }(D B)\right\} \\
\mathcal{P}_{G}^{\overline{D B}}= & \left\{p\left(t, x_{1}, x_{2}\right) \in \mathcal{P}_{G}:\left(T, X_{1}, X_{2}\right)\right. \text { do not } \\
& \text { satisfy }(D B)\}
\end{aligned}
$$

and

$$
\begin{aligned}
\mathcal{P}_{N G}^{D B}= & \left\{p\left(t, x_{1}, x_{2}\right) \in \mathcal{P}_{N G}:\left(T, X_{1}, X_{2}\right) \text { satisfy }(D B)\right\} \\
\mathcal{P}_{N G}^{\overline{D B}}= & \left\{p\left(t, x_{1}, x_{2}\right) \in \mathcal{P}_{N G}:\left(T, X_{1}, X_{2}\right)\right. \text { do not } \\
& \text { satisfy }(D B)\}
\end{aligned}
$$

Finally, we partition the set $\mathcal{P}_{N G}^{D B}$ into two disjoint sets $\mathcal{P}_{N G}^{D B(a)}$ and $\mathcal{P}_{N G}^{D B(b)}$ with $\mathcal{P}_{N G}^{D B}=\mathcal{P}_{N G}^{D B(a)} \cup \mathcal{P}_{N G}^{D B(b)}$, as

$$
\begin{aligned}
\mathcal{P}_{N G}^{D B(a)}= & \left\{p\left(t, x_{1}, x_{2}\right) \in \mathcal{P}_{N G}^{D B}:\right. \text { covariance matrix of } \\
& p\left(t, x_{1}, x_{2}\right) \text { is } Q \text { and there exists a } \mathcal{J G} \\
& \left(T_{G}, X_{1 G}, X_{2 G}\right) \text { with covariance matrix } Q \\
& \text { satisfying }(D B)\} \\
\mathcal{P}_{N G}^{D B(b)}= & \left\{p\left(t, x_{1}, x_{2}\right) \in \mathcal{P}_{N G}^{D B}:\right. \text { covariance matrix of } \\
& p\left(t, x_{1}, x_{2}\right) \text { is } Q \text { and there does not exist a } \mathcal{J G} \\
& \left(T_{G}, X_{1 G}, X_{2 G}\right) \text { with covariance matrix } Q \\
& \text { satisfying }(D B)\}
\end{aligned}
$$

So far, we have partitioned the set of input distributions into five disjoint sets: $\mathcal{P}_{G}^{D B}, \mathcal{P}_{G}^{\overline{D B}}, \mathcal{P}_{N G}^{D B(a)}, \mathcal{P}_{N G}^{D B(b)}$, and $\mathcal{P}_{N G}^{\overline{D B}}$. To visualize this partition of the set of input distributions, see Fig. 6 . It is clear that the input distributions which fall into the sets $\mathcal{P}_{G}^{\overline{D B}}$ and $\mathcal{P} \overline{N G}$ need not be considered since they do not satisfy the constraint $(D B)$ and do not have any consequence when evaluating our outer bounds. Therefore, we only need to restrict our attention on the three remaining sets $\mathcal{P}_{G}^{D B}, \mathcal{P}_{N G}^{D B(a)}$, and $\mathcal{P}_{N G}^{D B(b)}$, i.e., those input distributions which satisfy the dependence balance bound.

We explicitly evaluate our outer bound in the following three steps:

1) We first explicitly characterize the region of rate pairs provided by our outer bound for the probability distributions in the set $\mathcal{P}_{G}^{D B}$.

2) In the second step, we will show that for any input distribution belonging to the set $\mathcal{P}_{N G}^{D B(a)}$, there exists an input distribution in the set $\mathcal{P}_{G}^{D B}$ which yields a set of larger rate pairs. This leads to the conclusion that we do not need to consider the input distributions in the set $\mathcal{P}_{N G}^{D B(a)}$ in evaluating our outer bound.

3) We next focus on the set $\mathcal{P}_{N G}^{D B(b)}$ and show that for any nonGaussian input distribution $p\left(t, x_{1}, x_{2}\right) \in \mathcal{P}_{N G}^{D B(b)}$, we can construct a jointly Gaussian input distribution satisfying $(D B)$, i.e., we can find a corresponding input distribution in $\mathcal{P}_{G}^{D B}$, which yields a set of rates which includes the set of rates of the fixed non-Gaussian input distribution $p\left(t, x_{1}, x_{2}\right)$.

The main step in evaluating our outer bounds is step 3 described above. The proofs of step 3 for noisy feedback and user cooperation models are entirely different and do not follow from each other. The evaluation in step 1 is slightly different for all three settings, also owing to the channel models. Hence, we will separately focus on these models in the following three sections. Contrary to steps 1 and 3, step 2 is common for all channel models. Therefore, we first present the common result for all channel models here. In step 2, we consider any non-Gaussian input distribution $p\left(t, x_{1}, x_{2}\right)$ in $\mathcal{P}_{N G}^{D B(a)}$ with a covariance matrix $Q$. For such an input distribution, we know by the maximum entropy theorem [8], that the rates provided by a jointly Gaussian triple with the same covariance matrix $Q$ are always at least as large as the rates provided by the chosen non-Gaussian distribution. Therefore, for any input distribution in $\mathcal{P}_{N G}^{D B(a)}$, there always exists an input distribution in $\mathcal{P}_{G}^{D B}$, satisfying $(D B)$, which yields larger rates. This means that we can ignore the set $\mathcal{P}_{N G}^{D B(a)}$ altogether while evaluating our outer bounds.

To set the stage for our evaluations in steps 1 and 3 for the three channel models, let us define $\mathcal{Q}$ as the set of all valid $3 \times 3$ covariance matrices of three random variables $\left(T, X_{1}, X_{2}\right)$. A typical element $Q$ in the set $\mathcal{Q}$ takes the following form

$$
\begin{aligned}
Q & =E\left[\left(X_{1} X_{2} T\right)\left(X_{1} X_{2} T\right)^{T}\right] \\
& =\left(\begin{array}{ccc}
P_{1} & \rho_{12} \sqrt{P_{1} P_{2}} & \rho_{1 T} \sqrt{P_{1} P_{T}} \\
\rho_{12} \sqrt{P_{1} P_{2}} & P_{2} & \rho_{2 T} \sqrt{P_{2} P_{T}} \\
\rho_{1 T} \sqrt{P_{1} P_{T}} & \rho_{2 T} \sqrt{P_{2} P_{T}} & P_{T}
\end{array}\right) .
\end{aligned}
$$

A necessary condition for $Q$ to be a valid covariance matrix is that it is positive semi-definite, i.e., $\operatorname{det}(Q) \geq 0$ and $\rho_{12}, \rho_{1 T}, \rho_{2 T} \in[-1,+1]$. This implies

$$
\operatorname{det}(Q)=P_{1} P_{2} P_{T} \Delta \geq 0
$$

where we have defined for simplicity

$$
\Delta=1-\rho_{12}^{2}-\rho_{1 T}^{2}-\rho_{2 T}^{2}+2 \rho_{1 T} \rho_{2 T} \rho_{12} .
$$

\section{Evaluation of $\mathcal{D} \mathcal{B}_{N F}^{M A C}$}

In this section we explicitly evaluate Theorem 3 for the Gaussian MAC with noisy feedback described by (25)-(27) in Section VI. We start with step 1 . We consider an input distribution in $\mathcal{P}_{G}^{D B}$, i.e., a jointly Gaussian triple $\left(T_{G}, X_{1 G}, X_{2 G}\right)$ with a covariance matrix $Q$. Let us first characterize the set of rate constraints for this triple. It is straightforward to evaluate the three rate constraints appearing in (29)-(31) for this input distribution

$$
\begin{array}{r}
R_{1} \leq I\left(X_{1 G} ; Y \mid X_{2 G}, T_{G}\right)=\frac{1}{2} \log \left(1+\frac{f_{1}(Q)}{\sigma_{Z}^{2}}\right) \\
R_{2} \leq I\left(X_{2 G} ; Y \mid X_{1 G}, T_{G}\right)=\frac{1}{2} \log \left(1+\frac{f_{2}(Q)}{\sigma_{Z}^{2}}\right) \\
R_{1}+R_{2} \leq I\left(X_{1 G}, X_{2 G} ; Y \mid T_{G}\right)=\frac{1}{2} \log \left(1+\frac{f_{3}(Q)}{\sigma_{Z}^{2}}\right)
\end{array}
$$


where we have defined (63)-(65), shown at the bottom of the page. Finally, evaluating the constraint in (33), we conclude that this input distribution satisfies the constraint in (33) iff

$$
f_{3}(Q) \leq f_{1}(Q)+f_{2}(Q)+\frac{f_{1}(Q) f_{2}(Q)}{\left(\sigma_{Z}^{2}+\frac{\sigma_{Z_{1}}^{2} \sigma_{Z_{2}}^{2}}{\left(\sigma_{Z_{1}}^{2}+\sigma_{Z_{2}}^{2}\right)}\right)}
$$

To summarize, the set of rate pairs provided by an input distribution in $\mathcal{P}_{G}^{D B}$, with a covariance matrix $Q$, are given by those in (60)-(62), where $f_{i}(Q), i=1,2,3$, in those inequalities are subject to the constraint in (66). As we have discussed earlier, from evaluation of step 2 in Section IX, we know that all rate pairs contributed by input distributions in $\mathcal{P}_{N G}^{D B(a)}$ are covered by those given in $\mathcal{P}_{G}^{D B}$.

We now arrive at step 3 of our evaluation. Consider any input distribution $p\left(t, x_{1}, x_{2}\right)$ in $\mathcal{P}_{N G}^{D B(b)}$ with a covariance matrix $Q$. By the definition of the set $\mathcal{P}_{N G}^{D B(b)}$, we know that $Q$ does not satisfy (33), which implies

$$
f_{3}(Q)>f_{1}(Q)+f_{2}(Q)+\frac{f_{1}(Q) f_{2}(Q)}{\left(\sigma_{Z}^{2}+\frac{\sigma_{Z_{1}}^{2} \sigma_{Z_{2}}^{2}}{\left(\sigma_{Z_{1}}^{2}+\sigma_{Z_{2}}^{2}\right)}\right)} .
$$

We also note that for any $\left(T, X_{1}, X_{2}\right)$ with a covariance matrix $Q$

$$
\begin{aligned}
R_{1} & \leq I\left(X_{1} ; Y \mid X_{2}, T\right) \leq \frac{1}{2} \log \left(1+\frac{f_{1}(Q)}{\sigma_{Z}^{2}}\right) \\
R_{2} & \leq I\left(X_{2} ; Y \mid X_{1}, T\right) \leq \frac{1}{2} \log \left(1+\frac{f_{2}(Q)}{\sigma_{Z}^{2}}\right) \\
R_{1}+R_{2} & \leq I\left(X_{1}, X_{2} ; Y \mid T\right) \leq \frac{1}{2} \log \left(1+\frac{f_{3}(Q)}{\sigma_{Z}^{2}}\right)
\end{aligned}
$$

which is a simple consequence of the maximum entropy theorem [8]. Note that so far, we have not used the fact that the given non-Gaussian input distribution satisfies the dependence balance constraint in (33). We will now make use of this fact by rewriting (33) as follows:

$$
\begin{aligned}
0 \leq & I\left(X_{1} ; X_{2} \mid Y_{F_{1}}, Y_{F_{2}}, T\right)-I\left(X_{1} ; X_{2} \mid T\right) \\
= & I\left(X_{1} ; Y_{F_{1}}, Y_{F_{2}} \mid X_{2}, T\right)-I\left(X_{1} ; Y_{F_{1}}, Y_{F_{2}} \mid T\right) \\
= & h\left(Y_{F_{1}}, Y_{F_{2}} \mid X_{1}, T\right)+h\left(Y_{F_{1}}, Y_{F_{2}} \mid X_{2}, T\right) \\
& -h\left(Y_{F_{1}}, Y_{F_{2}} \mid T\right)-h\left(Y_{F_{1}}, Y_{F_{2}} \mid X_{1}, X_{2}, T\right)
\end{aligned}
$$

We express the above constraint as

$$
\begin{aligned}
h\left(Y_{F_{1}}, Y_{F_{2}} \mid T\right)+h\left(Y_{F_{1}}, Y_{F_{2}} \mid X_{1}, X_{2}, T\right) \\
\quad \leq h\left(Y_{F_{1}}, Y_{F_{2}} \mid X_{1}, T\right)+h\left(Y_{F_{1}}, Y_{F_{2}} \mid X_{2}, T\right)
\end{aligned}
$$

Before proceeding, we state a recently discovered multivariate generalization [16] of Costa's EPI [17].

Lemma 1: For any arbitrary random vector $\mathbf{Y} \in \mathbb{R}^{2}$, independent of $\mathbf{V} \in \mathbb{R}^{2}$, where $\mathbf{V}$ is a zero-mean, Gaussian random vector with each component having unit variance, the entropy power $N\left(\Lambda^{1 / 2} \mathbf{Y}+\mathbf{V}\right)$ is concave in $\Lambda$, where the entropy power is defined as

$$
N(\mathbf{Y})=\frac{1}{(2 \pi \mathrm{e})} \mathrm{e}^{h(\mathbf{Y})}
$$

and $\Lambda$ is a diagonal matrix with components $\left(\lambda_{1}, \lambda_{2}\right)$.

We can therefore write for any pair of diagonal matrices $\Lambda_{1}, \Lambda_{2}$ and for any $\mu \in[0,1]$,

$$
\begin{aligned}
& \mu N\left(\Lambda_{1}^{1 / 2} \mathbf{Y}+\mathbf{V}\right)+(1-\mu) N\left(\Lambda_{2}^{1 / 2} \mathbf{Y}+\mathbf{V}\right) \\
& \leq N\left(\left(\mu \Lambda_{1}+(1-\mu) \Lambda_{2}\right)^{1 / 2} \mathbf{Y}+\mathbf{V}\right)
\end{aligned}
$$

We start by obtaining a lower bound for the first term $h\left(Y_{F_{1}}, Y_{F_{2}} \mid T\right)$ in (74):

$$
\begin{aligned}
h\left(Y_{F_{1}}, Y_{F_{2}} \mid T\right) \\
=\int f_{T}(t) h\left(Y+Z_{1}, Y+Z_{2} \mid T=t\right) d t \\
\geq \int f_{T}(t) \frac{1}{2} \log \left((2 \pi \mathrm{e})^{2} \sigma_{Z_{1}}^{2} \sigma_{Z_{2}}^{2}\right. \\
\left.\quad+2 \pi \mathrm{e}\left(\sigma_{Z_{1}}^{2}+\sigma_{Z_{2}}^{2}\right) \mathrm{e}^{2 h(Y \mid T=t)}\right) d t \\
\geq \\
\quad \frac{1}{2} \log \left((2 \pi \mathrm{e})^{2} \sigma_{Z_{1}}^{2} \sigma_{Z_{2}}^{2}+2 \pi \mathrm{e}\left(\sigma_{Z_{1}}^{2}+\sigma_{Z_{2}}^{2}\right) \mathrm{e}^{2 h(Y \mid T)}\right)
\end{aligned}
$$

where (78) follows from the conditional version of Lemma 1, by selecting the following $\Lambda_{1}, \Lambda_{2}$ and $\mu$

$$
\Lambda_{1}=\left(\begin{array}{ll}
\kappa & 0 \\
0 & 0
\end{array}\right), \quad \Lambda_{2}=\left(\begin{array}{ll}
0 & 0 \\
0 & \kappa
\end{array}\right)
$$

where

$$
\kappa=\frac{\left(\sigma_{Z_{1}}^{2}+\sigma_{Z_{2}}^{2}\right)}{\sigma_{Z_{1}}^{2} \sigma_{Z_{2}}^{2}}
$$

and

$$
\mu=\frac{\sigma_{Z_{2}}^{2}}{\left(\sigma_{Z_{1}}^{2}+\sigma_{Z_{2}}^{2}\right)}
$$

and by making the following substitutions:

$$
V_{1}=\frac{Z_{1}}{\sigma_{Z_{1}}}, \quad V_{2}=\frac{Z_{2}}{\sigma_{Z_{2}}}
$$

$$
\begin{aligned}
f_{1}(Q) & =\operatorname{Var}\left(X_{1 G} \mid X_{2 G}, T_{G}\right)=\frac{\Delta P_{1}}{\left(1-\rho_{2 T}^{2}\right)} \\
f_{2}(Q) & =\operatorname{Var}\left(X_{2 G} \mid X_{1 G}, T_{G}\right)=\frac{\Delta P_{2}}{\left(1-\rho_{1 T}^{2}\right)} \\
f_{3}(Q) & =\operatorname{Var}\left(X_{1 G} \mid T_{G}\right)+\operatorname{Var}\left(X_{2 G} \mid T_{G}\right)+2 \operatorname{Cov}\left(X_{1 G}, X_{2 G} \mid T_{G}\right) \\
& =\left(1-\rho_{1 T}^{2}\right) P_{1}+\left(1-\rho_{2 T}^{2}\right) P_{2}+2\left(\rho_{12}-\rho_{1 T} \rho_{2 T}\right) \sqrt{P_{1} P_{2}}
\end{aligned}
$$


where $\mathbf{V}=\left[\begin{array}{ll}V_{1} & V_{2}\end{array}\right]^{T}$ and $\mathbf{Y}=\left[\begin{array}{ll}Y & Y\end{array}\right]^{T}$. A derivation of (78) is given in the Appendix. Next, (79) follows from the fact that $\log \left(\mathrm{e}^{x} c_{1}+c_{2}\right)$ is convex in $x$ for $c_{1}, c_{2} \geq 0$ and a subsequent application of Jensen's inequality $[8]^{1}$.

We next obtain an upper bound for the right hand side of (74) by using the maximum entropy theorem as

$$
\begin{aligned}
h\left(Y_{F_{1}}, Y_{F_{2}} \mid X_{1}, T\right)+h\left(Y_{F_{1}}, Y_{F_{2}} \mid X_{2}, T\right) \\
\leq \frac{1}{2} \log \left((2 \pi \mathrm{e})^{4}\left(f_{1}(Q)\left(\sigma_{Z_{1}}^{2}+\sigma_{Z_{2}}^{2}\right)+\eta\right) .\right. \\
\left.\quad\left(f_{2}(Q)\left(\sigma_{Z_{1}}^{2}+\sigma_{Z_{2}}^{2}\right)+\eta\right)\right)
\end{aligned}
$$

where we have defined

$$
\eta=\sigma_{Z_{1}}^{2} \sigma_{Z_{2}}^{2}+\sigma_{Z}^{2}\left(\sigma_{Z_{1}}^{2}+\sigma_{Z_{2}}^{2}\right) .
$$

Now, using (74), (79) and (84), we obtain an upper bound on $h(Y \mid T)$ as follows:

$$
h(Y \mid T) \leq \frac{1}{2} \log \left((2 \pi \mathrm{e})\left(\sigma_{Z}^{2}+f(Q)\right)\right)
$$

where we have defined for simplicity

$$
f(Q)=f_{1}(Q)+f_{2}(Q)+\frac{f_{1}(Q) f_{2}(Q)}{\left(\sigma_{Z}^{2}+\frac{\sigma_{Z_{1}}^{2} \sigma_{Z_{2}}^{2}}{\left(\sigma_{Z_{1}}^{2}+\sigma_{Z_{2}}^{2}\right)}\right) .}
$$

Using (86), we obtain an upper bound on the sum-rate $I\left(X_{1}, X_{2} ; Y \mid T\right)$ for any non-Gaussian distribution in $\mathcal{P}_{N G}^{D B(b)}$ as,

$$
R_{1}+R_{2} \leq I\left(X_{1}, X_{2} ; Y \mid T\right) \leq \frac{1}{2} \log \left(1+\frac{f(Q)}{\sigma_{Z}^{2}}\right) .
$$

Comparing with (70) and using the fact that $Q$ satisfies (67), i.e., $f(Q)<f_{3}(Q)$, we have the following set of inequalities

$$
\begin{aligned}
R_{1}+R_{2} & \leq I\left(X_{1}, X_{2} ; Y \mid T\right) \\
& \leq \frac{1}{2} \log \left(1+\frac{f(Q)}{\sigma_{Z}^{2}}\right) \\
& <\frac{1}{2} \log \left(1+\frac{f_{3}(Q)}{\sigma_{Z}^{2}}\right) .
\end{aligned}
$$

This leads to the observation that a combined application of the EPI and the dependence balance bound yields a strictly smaller upper bound for $I\left(X_{1}, X_{2} ; Y \mid T\right)$ for any distribution in $\mathcal{P}_{N G}^{D B(b)}$ than the one provided by the maximum entropy theorem. Therefore, the rate pairs contributed by an input distribution in $\mathcal{P}_{N G}^{D B(b)}$ with a covariance matrix $Q$ are always included in the set of rate pairs expressed by (68), (69) and (88), where $f(Q)$ is defined in (87).

We now arrive at the final step of our evaluation where we will show that for this input distribution in $\mathcal{P}_{N G}^{D B(b)}$, we can always find an input distribution in $\mathcal{P}_{G}^{D B}$, with a set of rate pairs which

\footnotetext{
${ }^{1}$ We should remark here, that an application of the regular form of vector EPI yields the following trivial lower bound on $h\left(Y_{F_{1}}, Y_{F_{2}} \mid T\right)$ and therefore, the new EPI is crucial for this step:
}

$$
h\left(Y_{F_{1}}, Y_{F_{2}} \mid T\right) \geq \frac{1}{2} \log \left((2 \pi \mathrm{e})^{2} \sigma_{Z_{1}}^{2} \sigma_{Z_{2}}^{2}\right)
$$

include the set of rate pairs expressed by (68), (69), and (88). In particular, we will show the existence of a valid covariance matrix $S$ for which the following inequalities hold true:

$$
\begin{aligned}
f_{1}(Q) & \leq f_{1}(S) \\
f_{2}(Q) & \leq f_{2}(S) \\
f(Q) & \leq f_{3}(S)
\end{aligned}
$$

and

$$
f_{3}(S)=f_{1}(S)+f_{2}(S)+\frac{f_{1}(S) f_{2}(S)}{\left(\sigma_{Z}^{2}+\frac{\sigma_{Z_{1}}^{2} \sigma_{Z_{2}}^{2}}{\left(\sigma_{Z_{1}}^{2}+\sigma_{Z_{2}}^{2}\right)}\right)} .
$$

Inequalities in (90)-(92) will guarantee that a Gaussian input distribution with covariance matrix $S$ yields a larger set of rate pairs than the set of rate pairs expressed by (68), (69) and (88) and the equality in (93) guarantees that this input distribution satisfies the dependence balance constraint with equality, hence it is a member of the set $\mathcal{P}_{G}^{D B}$.

Before showing the existence of such an $S$, we first characterize the set of covariance matrices $Q$ which satisfy (67). First recall that for any $Q$ to be a valid covariance matrix, we had the condition $\operatorname{det}(Q) \geq 0$ which is equivalent to $\Delta \geq 0$, which amounts to

$$
1-\rho_{12}^{2}-\rho_{1 T}^{2}-\rho_{2 T}^{2}+2 \rho_{1 T} \rho_{2 T} \rho_{12} \geq 0 .
$$

In particular, it is easy to verify that for any given fixed pair $\left(\rho_{1 T}, \rho_{2 T}\right) \in[-1,1] \times[-1,1]$, the set of $\rho_{12}$ which yield a valid $Q$ are such that

$$
\rho_{1 T} \rho_{2 T}-\lambda \leq \rho_{12} \leq \rho_{1 T} \rho_{2 T}+\lambda
$$

where we have defined

$$
\lambda=\sqrt{\left(1-\rho_{1 T}^{2}\right)\left(1-\rho_{2 T}^{2}\right)} .
$$

We now consider two cases which can arise for a given covariance matrix $Q$.

Case 1. $Q$ is such that $\rho_{12}=\rho_{1 T} \rho_{2 T}-\alpha$, for some $\alpha \in[0, \lambda]$ : This case is rather trivial and the following simple choice of $S$ works:

$$
\begin{aligned}
& \rho_{1 T}^{(S)}=\rho_{1 T}, \quad \rho_{2 T}^{(S)}=\rho_{2 T} \\
& \rho_{12}^{(S)}=\rho_{1 T} \rho_{2 T} .
\end{aligned}
$$

Clearly, this $S$ satisfies the dependence balance bound. Moreover, the following inequalities hold as well

$$
\begin{aligned}
f_{1}(Q) & \leq f_{1}(S)=\left(1-\rho_{1 T}^{2}\right) P_{1} \\
f_{2}(Q) & \leq f_{2}(S)=\left(1-\rho_{2 T}^{2}\right) P_{2} \\
f(Q) & <f_{3}(Q) \\
& =\left(1-\rho_{1 T}^{2}\right) P_{1}+\left(1-\rho_{2 T}^{2}\right) P_{2}-2 \alpha \sqrt{P_{1} P_{2}} \\
& \leq\left(1-\rho_{1 T}^{2}\right) P_{1}+\left(1-\rho_{2 T}^{2}\right) P_{2} \\
& =f_{3}(S) .
\end{aligned}
$$


Case 2. $Q$ is such that $\rho_{12}=\rho_{1 T} \rho_{2 T}+\alpha_{0}$, for some $\alpha_{0} \in$ $(0, \lambda]$ and $Q$ satisfies (67): For this case, we will construct a valid covariance matrix $S$ as follows:

$$
\begin{aligned}
& \rho_{1 T}^{(S)}=\rho_{1 T}, \quad \rho_{2 T}^{(S)}=\rho_{2 T} \\
& \rho_{12}^{(S)}=\rho_{1 T} \rho_{2 T}+\alpha^{*}, \quad \text { for some } 0<\alpha^{*}<\alpha_{0} .
\end{aligned}
$$

We define a parameterized covariance matrix $Q(\alpha)$ with entries

$$
\begin{aligned}
& \rho_{1 T}(\alpha)=\rho_{1 T}, \quad \rho_{2 T}(\alpha)=\rho_{2 T} \\
& \rho_{12}(\alpha)=\rho_{1 T} \rho_{2 T}+\alpha
\end{aligned}
$$

where $0 \leq \alpha \leq \alpha_{0}$. We now define a function of the parameter $\alpha$ of a valid covariance matrix $Q(\alpha)$ as

$$
\begin{aligned}
g(\alpha)= & f_{1}(Q(\alpha))+f_{2}(Q(\alpha))+\frac{f_{1}(Q(\alpha)) f_{2}(Q(\alpha))}{\left(\sigma_{Z}^{2}+\frac{\sigma_{Z_{1}}^{2} \sigma_{Z_{2}}^{2}}{\left(\sigma_{Z_{1}}^{2}+\sigma_{Z_{2}}^{2}\right)}\right)} \\
& -f_{3}(Q(\alpha)) \\
= & f(Q(\alpha))-f_{3}(Q(\alpha)) .
\end{aligned}
$$

Now note the fact that

$$
g(0)=\frac{\left(1-\rho_{1 T}^{2}\right)\left(1-\rho_{2 T}^{2}\right) P_{1} P_{2}}{\left(\sigma_{Z}^{2}+\frac{\sigma_{Z_{1}}^{2} \sigma_{Z_{2}}^{2}}{\left(\sigma_{Z_{1}}^{2}+\sigma_{Z_{2}}^{2}\right)}\right)}>0 .
$$

We are also given that $Q$ satisfies (67) for some $\alpha_{0}$, which implies that,

$$
g\left(\alpha_{0}\right)<0 .
$$

Now, we take the first derivative of the function $g(\alpha)$, to obtain

$$
\begin{aligned}
& \frac{d g(\alpha)}{d \alpha} \\
& \quad=-2 \alpha\left(\frac{P_{1}}{\left(1-\rho_{2 T}^{2}\right)}+\frac{P_{2}}{\left(1-\rho_{1 T}^{2}\right)}\right) \\
& \quad-\frac{4 \alpha P_{1} P_{2}}{\left(\sigma_{Z}^{2}+\frac{\sigma_{Z_{1}}^{2} \sigma_{Z_{2}}^{2}}{\left(\sigma_{Z_{1}}^{2}+\sigma_{Z_{2}}^{2}\right)}\right)}\left(1-\left(\frac{\alpha}{\lambda}\right)^{2}\right)-2 \sqrt{P_{1} P_{2}} \\
& \quad \leq 0
\end{aligned}
$$

which implies that $g(\alpha)$ is monotonically decreasing in $\alpha$. This implies that there exists an $\alpha^{*} \in\left(0, \alpha_{0}\right)$ such that $g\left(\alpha^{*}\right)=0^{2}$. We use this $\alpha^{*}$ to construct our new covariance matrix $S$ as follows:

$$
\begin{aligned}
& \rho_{1 T}^{(S)}=\rho_{1 T}, \quad \rho_{2 T}^{(S)}=\rho_{2 T} \\
& \rho_{12}^{(S)}=\rho_{1 T} \rho_{2 T}+\alpha^{*} .
\end{aligned}
$$

\footnotetext{
${ }^{2}$ We should remark here that the existence of an $\alpha^{*} \in\left(0, \alpha_{0}\right)$, with $g\left(\alpha^{*}\right)=$ 0 can also be proved alternatively by invoking the mean value theorem, since we have $g(0)>0, g\left(\alpha_{0}\right)<0$ and $g(\alpha)$ is a continuous function of $\alpha$. Monotonicity of $g(\alpha)$ in fact proves a stronger statement that such an $\alpha^{*}$ exists and is also unique.
}

It now remains to check wether $S$ satisfies the four conditions in (90)-(93). The condition (93) is met with equality, since we have $g\left(\alpha^{*}\right)=0$. Moreover, $f_{1}(Q)=f_{1}\left(Q\left(\alpha_{0}\right)\right) \leq f_{1}\left(Q\left(\alpha^{*}\right)\right)=$ $f_{1}(S)$ since $f_{1}(Q(\alpha))$ is monotonically decreasing in $\alpha$ for $\alpha \in$ $[0, \lambda]$. Similarly, we also have $f_{2}(Q) \leq f_{2}(S)$. Finally

$$
\begin{aligned}
f(Q) & =f_{1}(Q)+f_{2}(Q)+\frac{f_{1}(Q) f_{2}(Q)}{\left(\sigma_{Z}^{2}+\frac{\sigma_{Z_{1}}^{2} \sigma_{Z_{2}}^{2}}{\left(\sigma_{Z_{1}}^{2}+\sigma_{Z_{2}}^{2}\right)}\right)} \\
& \leq f_{1}(S)+f_{2}(S)+\frac{f_{1}(S) f_{2}(S)}{\left(\sigma_{Z}^{2}+\frac{\sigma_{Z_{1}}^{2} \sigma_{Z_{2}}^{2}}{\left(\sigma_{Z_{1}}^{2}+\sigma_{Z_{2}}^{2}\right)}\right)} \\
& =f_{3}(S) .
\end{aligned}
$$

This shows the existence of a valid covariance matrix $S$ which satisfies (33) and yields a set of rates which includes the set of rates of the given non-Gaussian distribution with the covariance matrix $Q$.

Above two cases show that for any non-Gaussian distribution $p\left(t, x_{1}, x_{2}\right)$ in the set $\mathcal{P}_{N G}^{D B(b)}$, we can always find a jointly Gaussian triple $\left(T_{G}, X_{1 G}, X_{2 G}\right)$ in $\mathcal{P}_{G}^{D B}$ that yields a set of rates subsuming the set of rates of the given non-Gaussian distribution. This consequently completes the proof of the statement that it is sufficient to consider jointly Gaussian $\left(T, X_{1}, X_{2}\right)$ in $\mathcal{P}_{G}^{D B}$ when evaluating our outer bound.

The dependence balance based outer bound can now be written in an explicit form as follows:

$$
\begin{aligned}
\mathcal{D B} \mathcal{B}_{N F}^{M A C}=\bigcup_{Q \in \mathcal{Q}^{D B}} & \left\{\left(R_{1}, R_{2}\right):\right. \\
& R_{1} \leq \frac{1}{2} \log \left(1+\frac{f_{1}(Q)}{\sigma_{Z}^{2}}\right) \\
& R_{2} \leq \frac{1}{2} \log \left(1+\frac{f_{2}(Q)}{\sigma_{Z}^{2}}\right) \\
& \left.R_{1}+R_{2} \leq \frac{1}{2} \log \left(1+\frac{f_{3}(Q)}{\sigma_{Z}^{2}}\right)\right\}
\end{aligned}
$$

where $\mathcal{Q}^{D B}$ is the set of $3 \times 3$ covariance matrices of the form (57) satisfying,

$$
f_{3}(Q) \leq f_{1}(Q)+f_{2}(Q)+\frac{f_{1}(Q) f_{2}(Q)}{\left(\sigma_{Z}^{2}+\frac{\sigma_{Z_{1}}^{2} \sigma_{Z_{2}}^{2}}{\left(\sigma_{Z_{1}}^{2}+\sigma_{Z_{2}}^{2}\right)}\right)}
$$

where

$$
\begin{aligned}
f_{1}(Q)= & \frac{\Delta P_{1}}{\left(1-\rho_{2 T}^{2}\right)} \\
f_{2}(Q)= & \frac{\Delta P_{2}}{\left(1-\rho_{1 T}^{2}\right)} \\
f_{3}(Q)= & \left(1-\rho_{1 T}^{2}\right) P_{1}+\left(1-\rho_{2 T}^{2}\right) P_{2} \\
& +2\left(\rho_{12}-\rho_{1 T} \rho_{2 T}\right) \sqrt{P_{1} P_{2}},
\end{aligned}
$$

and

$$
\Delta=1-\rho_{1 T}^{2}-\rho_{2 T}^{2}-\rho_{12}^{2}+2 \rho_{1 T} \rho_{2 T} \rho_{12}
$$

where $\rho_{12}, \rho_{1 T}$ and $\rho_{2 T}$ are all in $[-1,1]$. 
The cut-set outer bound given in (1)-(4) is evaluated for the Gaussian MAC with noisy feedback described in (25)-(27) as

$$
\begin{aligned}
\mathcal{C} \mathcal{S}_{N F}^{M A C}= & \bigcup_{\rho \in[0,1]}\left\{\left(R_{1}, R_{2}\right):\right. \\
& R_{1} \leq \frac{1}{2} \log \left(1+\frac{\left(1-\rho^{2}\right) P_{1}}{\sigma_{Z}^{2}}\right) \\
& R_{2} \leq \frac{1}{2} \log \left(1+\frac{\left(1-\rho^{2}\right) P_{2}}{\sigma_{Z}^{2}}\right) \\
& \left.R_{1}+R_{2} \leq \frac{1}{2} \log \left(1+\frac{P_{1}+P_{2}+2 \rho \sqrt{P_{1} P_{2}}}{\sigma_{Z}^{2}}\right)\right\}
\end{aligned}
$$

We briefly mention what our outer bound gives for the the two limiting values of the backward noise variances $\sigma_{Z_{1}}^{2}$ and $\sigma_{Z_{2}}^{2}$.

1) $\sigma_{Z_{1}}^{2}, \sigma_{Z_{2}}^{2} \rightarrow 0$ : this case corresponds to the Gaussian MAC with noiseless feedback and the constraint (119) simplifies to

$$
f_{3}(Q) \leq f_{1}(Q)+f_{2}(Q)+\frac{f_{1}(Q) f_{2}(Q)}{\sigma_{Z}^{2}}
$$

which is simply stating that the sum-rate constraint should be at most as large as the sum of the individual rate constraints, i.e., another equivalent way of writing is

$$
\begin{aligned}
\frac{1}{2} \log \left(1+\frac{f_{3}(Q)}{\sigma_{Z}^{2}}\right) \leq & \frac{1}{2} \log \left(1+\frac{f_{1}(Q)}{\sigma_{Z}^{2}}\right) \\
& +\frac{1}{2} \log \left(1+\frac{f_{2}(Q)}{\sigma_{Z}^{2}}\right) .
\end{aligned}
$$

This is the same constraint as obtained by Ozarow in [10], and our outer bound coincides with the cut-set bound and yields the capacity region of the Gaussian MAC with noiseless feedback.

2) $\sigma_{Z_{1}}^{2}, \sigma_{Z_{2}}^{2} \rightarrow \infty$ : this case corresponds to very noisy feedback and our outer bound should collapse to the no-feedback capacity region of the Gaussian MAC. For this case, the constraint (119) simplifies to

$$
f_{3}(Q) \leq f_{1}(Q)+f_{2}(Q) .
$$

On substituting the values of $f_{1}(Q), f_{2}(Q)$ and $f_{3}(Q)$ in the above inequality, we obtain

$$
\begin{aligned}
& \left(\rho_{12}-\rho_{1 T} \rho_{2 T}\right) \\
& \quad \leq \frac{\left(\left(1-\rho_{1 T}^{2}\right) P_{1}+\left(1-\rho_{2 T}^{2}\right) P_{2}\right)}{2 \sqrt{P_{1} P_{2}}}\left(\frac{\Delta}{\lambda^{2}}-1\right) \\
& \leq 0
\end{aligned}
$$

where the last inequality comes from the fact that for any valid covariance matrix, $\Delta \leq \lambda^{2}$. This implies that the dependence balance bound only allows such covariance matrices $Q$ for which $\rho_{12} \leq \rho_{1 T} \rho_{2 T}$. But we know already from (97)-(98) that we can always find an $S$ for which we can select $\rho_{12}^{(S)}=\rho_{1 T} \rho_{2 T}$, which satisfies the dependence balance bound and yields larger rates than any $Q$ with $\rho_{12}<\rho_{1 T} \rho_{2 T}$. Thus, we only need to restrict our attention to those matrices $Q$ for which $\rho_{12}=\rho_{1 T} \rho_{2 T}$.
Such covariance matrices $Q$ correspond to those jointly Gaussian triples which satisfy the Markov chain $X_{1} \rightarrow$ $T \rightarrow X_{2}$. This can be observed by noting that for any jointly Gaussian $\left(T, X_{1}, X_{2}\right)$, with a covariance matrix $Q$, the condition $I\left(X_{1} ; X_{2} \mid T\right)=0$ holds iff $\operatorname{Var}\left(X_{1} \mid T\right)=$ $\operatorname{Var}\left(X_{1} \mid X_{2}, T\right)$, which is equivalent to $\rho_{12}=\rho_{1 T} \rho_{2 T}$. Proof of this statement is immediate by noting that for a jointly Gaussian triple, we have

$$
I\left(X_{1} ; X_{2} \mid T\right)=\frac{1}{2} \log \left(\frac{\operatorname{Var}\left(X_{1} \mid T\right)}{\operatorname{Var}\left(X_{1} \mid X_{2}, T\right)}\right) .
$$

Therefore, $T$ can be interpreted simply as a timesharing random variable and our outer bound yields the capacity region of the Gaussian MAC without feedback.

Figs. 7 and 8 illustrate $\mathcal{D} \mathcal{B}_{N F}^{M A C}$, the cut-set bound and the capacity region without feedback for the cases when $\sigma_{Z_{1}}^{2}=$ $\sigma_{Z_{2}}^{2}=2,5$ and 10 , where $P_{1}=P_{2}=\sigma_{Z}^{2}=1$. Figs. 9 and 10 illustrate $\mathcal{D} \mathcal{B}_{N F}^{M A C}$, the cut-set bound, the capacity region without feedback and an achievable rate region based on superposition coding [3] for the case when $\sigma_{Z_{1}}^{2}=\sigma_{Z_{2}}^{2}=0.3$ and $P_{1}=P_{2}=\sigma_{Z}^{2}=1$.

\section{A. Remark}

For the special case of Gaussian MAC with common, noisy feedback, where

$$
Y_{F_{1}}=Y_{F_{2}}=Y+V
$$

the evaluation of $\mathcal{D} \mathcal{B}_{N F}^{M A C}$ follows in a similar manner as in the case of different noisy feedback signals. The only difference arises in the application of the EPI. In particular, the regular EPI [8] suffices to provide a nontrivial upper bound on $I\left(X_{1}, X_{2} ; Y \mid T\right)$ than the one provided by the maximum entropy theorem [8]. The remainder of the proof of evaluation of our outer bound for this channel model follows along the same lines as the proof for different noisy feedback signals. The final expressions of outer bounds for these two channel models only differ over the constraint (119). For the case of common, noisy feedback, the set $\mathcal{Q}^{D B}$ comprises of $3 \times 3$ covariance matrices of the form (57) satisfying

$$
f_{3}(Q) \leq f_{1}(Q)+f_{2}(Q)+\frac{f_{1}(Q) f_{2}(Q)}{\left(\sigma_{Z}^{2}+\sigma_{V}^{2}\right)} .
$$

Now consider the Gaussian MAC with different noisy feedback signals $Y_{F_{1}}$ and $Y_{F_{2}}$ at the transmitters 1 and 2, respectively. If the variances of feedback noises $Z_{1}$ and $Z_{2}$ are such that, $\sigma_{Z_{1}}^{2}=\sigma_{Z_{2}}^{2}=\sigma_{V}^{2}$, then the dependence balance constraint (119) simplifies as

$$
f_{3}(Q) \leq f_{1}(Q)+f_{2}(Q)+\frac{f_{1}(Q) f_{2}(Q)}{\left(\sigma_{Z}^{2}+\frac{\sigma_{V}^{2}}{2}\right)}
$$

This implies that if a covariance matrix $Q$ satisfies the constraint (132), then it also satisfies (133) but the converse statement may not always be true. This means that the resulting outer bound for the Gaussian MAC with common noisy feedback, with feedback noise variance $\sigma_{V}^{2}$ can be strictly smaller than the resulting outer bound for Gaussian MAC with different noisy feedback signals, when the feedback noise variances are $\sigma_{Z_{1}}^{2}=\sigma_{Z_{2}}^{2}=\sigma_{V}^{2}$. 


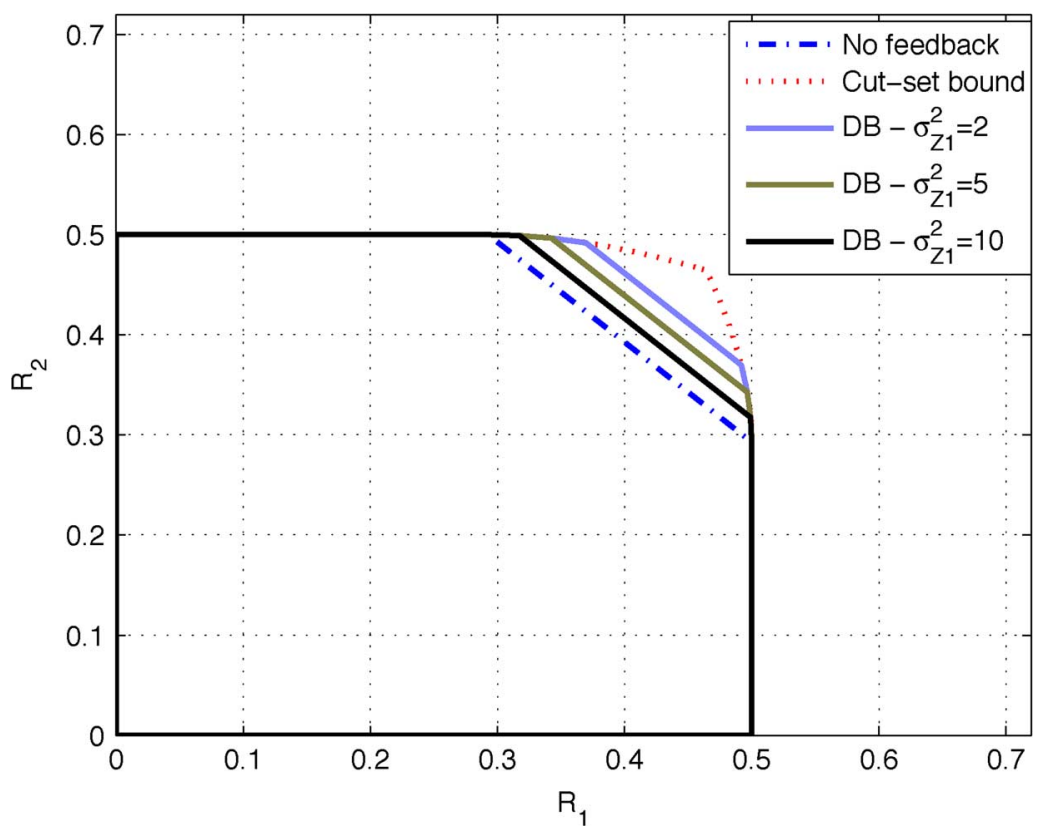

Fig. 7. Illustration of outer bounds for $P_{1}=P_{2}=\sigma_{Z}^{2}=1$ and $\sigma_{Z_{1}}^{2}=\sigma_{Z_{2}}^{2}=2,5,10$.

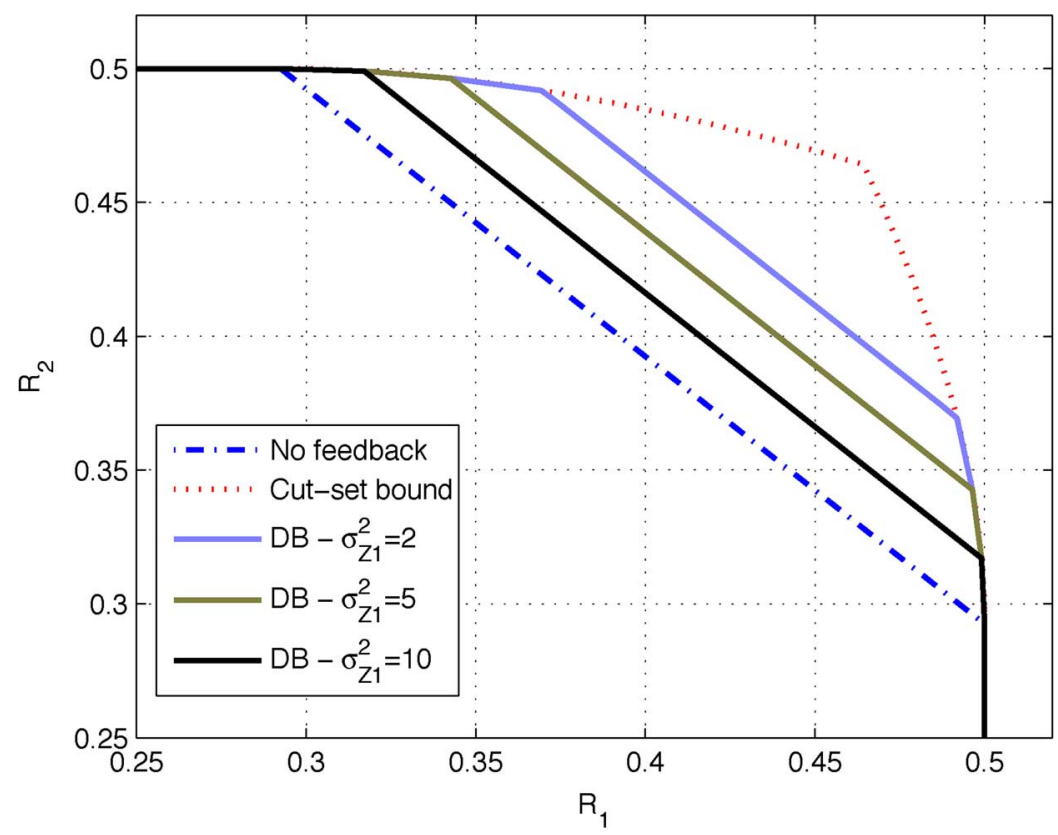

Fig. 8. Enlarged illustration of Fig. 7.

\section{Evaluation of $\mathcal{D} \mathcal{B}_{U C}^{M A C}$}

In this section, we will explicitly evaluate Theorem 4 for the Gaussian MAC with user cooperation described by (34)-(36) in Section VII. We start with step 1 and characterize the set of jointly Gaussian triples $\left(T_{G}, X_{1 G}, X_{2 G}\right)$ in $\mathcal{P}_{G}^{D B}$. For this purpose, we rewrite (43) as follows:

$$
\begin{aligned}
0 \leq & I\left(X_{1} ; X_{2} \mid Y_{F_{1}}, Y_{F_{2}}, T\right)-I\left(X_{1} ; X_{2} \mid T\right) \\
= & I\left(X_{1} ; Y_{F_{1}}, Y_{F_{2}} \mid X_{2}, T\right)-I\left(X_{1} ; Y_{F_{1}}, Y_{F_{2}} \mid T\right) \\
= & h\left(Y_{F_{1}}, Y_{F_{2}} \mid X_{1}, T\right)+h\left(Y_{F_{1}}, Y_{F_{2}} \mid X_{2}, T\right) \\
& -h\left(Y_{F_{1}}, Y_{F_{2}} \mid T\right)-h\left(Y_{F_{1}}, Y_{F_{2}} \mid X_{1}, X_{2}, T\right)
\end{aligned}
$$

and express the above constraint as follows:

$$
\begin{aligned}
& h\left(Y_{F_{1}}, Y_{F_{2}} \mid T\right)+h\left(Y_{F_{1}}, Y_{F_{2}} \mid X_{1}, X_{2}, T\right) \\
& \quad \leq h\left(Y_{F_{1}}, Y_{F_{2}} \mid X_{1}, T\right)+h\left(Y_{F_{1}}, Y_{F_{2}} \mid X_{2}, T\right) .
\end{aligned}
$$

Making use of the following equalities (see (138)-(140), shown at the bottom of the next page): we obtain a simplified expression for (137) as

$$
h\left(Y_{F_{1}}, Y_{F_{2}} \mid T\right) \leq h\left(Y_{F_{1}} \mid X_{1}, T\right)+h\left(Y_{F_{2}} \mid X_{2}, T\right) .
$$

We further simplify (141) as (142)-(146), shown at the bottom of the next page, where (145) follows from the Markov chain 
$Y_{F_{1}} \rightarrow X_{2} \rightarrow\left(T, Y_{F_{2}}\right)$. Therefore, the dependence balance constraint in (43) is equivalent to following two equalities:

$$
\begin{aligned}
I\left(Y_{F_{1}} ; X_{1} \mid T\right) & =0 \\
I\left(Y_{F_{2}} ; X_{2} \mid Y_{F_{1}}, T\right) & =0 .
\end{aligned}
$$

Next, we show that if any jointly Gaussian triple $\left(T, X_{1}, X_{2}\right)$ satisfies the constraints (147)-(148) then it satisfies the Markov chain $X_{1} \rightarrow T \rightarrow X_{2}$. Conversely, we will show that if any jointly Gaussian triple $\left(T, X_{1}, X_{2}\right)$ satisfies $X_{1} \rightarrow T \rightarrow X_{2}$, then it satisfies (147)-(148).

We start by evaluating (147) and (148) for a jointly Gaussian $\left(T_{G}, X_{1 G}, X_{2 G}\right)$ which is equivalent to

$$
\begin{aligned}
& 0=I\left(\sqrt{h_{21}} X_{2 G}+Z_{1} ; X_{1 G} \mid T_{G}\right) \\
& 0=I\left(\sqrt{h_{12}} X_{1 G}+Z_{2} ; X_{2 G} \mid \sqrt{h_{21}} X_{2 G}+Z_{1}, T_{G}\right)
\end{aligned}
$$

These equalities are equivalent to

$$
\operatorname{Cov}\left(X_{1 G}, X_{2 G} \mid T_{G}\right)=0 .
$$

Using the same argument as in (130), we obtain the following condition

$$
\rho_{12}=\rho_{1 T} \rho_{2 T} .
$$

This implies that a jointly Gaussian triple satisfies (147)-(148) iff $\rho_{12}=\rho_{1 T} \rho_{2 T}$.

On the other hand, consider any jointly Gaussian triple $\left(T_{G}, X_{1 G}, X_{2 G}\right)$, with a covariance matrix $Q$ which satisfies the Markov chain $X_{1 G} \rightarrow T_{G} \rightarrow X_{2 G}$. This is equivalent to $I\left(X_{1 G} ; X_{2 G} \mid T_{G}\right)=0$, which is equivalent to

$$
\rho_{12}=\rho_{1 T} \rho_{2 T} .
$$

This implies that if a jointly Gaussian triple $\left(T, X_{1}, X_{2}\right)$ satisfies the Markov chain $X_{1} \rightarrow T \rightarrow X_{2}$, then it satisfies (153) and therefore it also satisfies (147)-(148) and vice versa. As a consequence, we have explicitly characterized the set $\mathcal{P}_{G}^{D B}$, i.e., it comprises of only such jointly Gaussian distributions, $\left(T_{G}, X_{1 G}, X_{2 G}\right)$, for which $X_{1 G} \rightarrow T_{G} \rightarrow X_{2 G}$.

We can now write the set of rate pairs provided by our outer bound for a jointly Gaussian triple $\left(T_{G}, X_{1 G}, X_{2 G}\right)$ in the set $\mathcal{P}_{G}^{D B}$ as

$$
\begin{aligned}
R_{1} & \leq I\left(X_{1 G} ; Y, Y_{F_{2}} \mid X_{2 G}, T_{G}\right) \\
R_{2} & \leq I\left(X_{2 G} ; Y, Y_{F_{1}} \mid X_{1 G}, T_{G}\right) \\
R_{1}+R_{2} & \leq I\left(X_{1 G}, X_{2 G} ; Y, Y_{F_{1}}, Y_{F_{2}} \mid T_{G}\right) \\
R_{1}+R_{2} & \leq I\left(X_{1 G}, X_{2 G} ; Y\right)
\end{aligned}
$$

where $\left(T_{G}, X_{1 G}, X_{2 G}\right)$ satisfies the Markov chain $X_{1 G} \rightarrow$ $T_{G} \rightarrow X_{2 G}$. Moreover, from the evaluation of step 2 in Section IX, we know that all rate pairs contributed by input distributions in $\mathcal{P}_{N G}^{D B(a)}$ are covered by those given in $\mathcal{P}_{G}^{D B}$. Therefore, we do not need to consider the set $\mathcal{P}_{N G}^{D B(a)}$ in evaluating our outer bound.

We now arrive at step 3 of the evaluation of our outer bound where we will show that for any non-Gaussian input distribution $p\left(t, x_{1}, x_{2}\right) \in \mathcal{P}_{N G}^{D B(b)}$, we can always find an input distribution in $\mathcal{P}_{G}^{D B}$, with a set of rate pairs which include the set of rate pairs of the fixed non-Gaussian input distribution $p\left(t, x_{1}, x_{2}\right)$. Consider any triple $\left(T, X_{1}, X_{2}\right)$ with a non-Gaussian input distribution $p\left(t, x_{1}, x_{2}\right) \in \mathcal{P}_{N G}^{D B(b)}$, with a valid covariance matrix $Q$. By the definition of the set $\mathcal{P}_{N G}^{D B(b)}$, and as a consequence of (152), this covariance matrix has the property that $\rho_{12} \neq$ $\rho_{1 T} \rho_{2 T}$. Moreover, this non-Gaussian distribution satisfies the dependence balance bound, i.e., it satisfies (147) and (148). For our purpose, we only need (147). Since $I\left(Y_{F_{1}} ; X_{1} \mid T\right)=0$, this implies (see (158)-(159), shown at the bottom of the page). On the other hand, we also have $E\left[\left(\sqrt{h_{21}} X_{2}+Z_{1}\right) X_{1} \mid T\right]=$ $\sqrt{h_{21}} E\left[X_{1} X_{2} \mid T\right]$, which implies

$$
E\left[X_{1} X_{2} \mid T\right]=E\left[X_{2} \mid T\right] E\left[X_{1} \mid T\right]
$$

$$
\begin{aligned}
h\left(Y_{F_{1}}, Y_{F_{2}} \mid X_{1}, X_{2}, T\right) & =\frac{1}{2} \log \left((2 \pi \mathrm{e})^{2} \sigma_{Z_{1}}^{2} \sigma_{Z_{2}}^{2}\right) \\
h\left(Y_{F_{1}}, Y_{F_{2}} \mid X_{1}, T\right) & =\frac{1}{2} \log \left((2 \pi \mathrm{e}) \sigma_{Z_{2}}^{2}\right)+h\left(Y_{F_{1}} \mid X_{1}, T\right) \\
h\left(Y_{F_{1}}, Y_{F_{2}} \mid X_{2}, T\right) & =\frac{1}{2} \log \left((2 \pi \mathrm{e}) \sigma_{Z_{1}}^{2}\right)+h\left(Y_{F_{2}} \mid X_{2}, T\right)
\end{aligned}
$$

$$
\begin{aligned}
0 & \leq h\left(Y_{F_{1}} \mid X_{1}, T\right)+h\left(Y_{F_{2}} \mid X_{2}, T\right)-h\left(Y_{F_{1}}, Y_{F_{2}} \mid T\right) \\
& =h\left(Y_{F_{1}} \mid X_{1}, T\right)+h\left(Y_{F_{2}} \mid X_{2}, T\right)-h\left(Y_{F_{1}} \mid T\right)-h\left(Y_{F_{2}} \mid Y_{F_{1}}, T\right) \\
& =-I\left(Y_{F_{1}} ; X_{1} \mid T\right)+h\left(Y_{F_{2}} \mid X_{2}, T\right)-h\left(Y_{F_{2}} \mid Y_{F_{1}}, T\right) \\
& =-I\left(Y_{F_{1}} ; X_{1} \mid T\right)+h\left(Y_{F_{2}} \mid X_{2}, Y_{F_{1}}, T\right)-h\left(Y_{F_{2}} \mid Y_{F_{1}}, T\right) \\
& =-I\left(Y_{F_{1}} ; X_{1} \mid T\right)-I\left(Y_{F_{2}} ; X_{2} \mid Y_{F_{1}}, T\right)
\end{aligned}
$$

$$
\begin{aligned}
E\left[\left(\sqrt{h_{21}} X_{2}+Z_{1}\right) X_{1} \mid T\right] & =E\left[\sqrt{h_{21}} X_{2}+Z_{1} \mid T\right] E\left[X_{1} \mid T\right] \\
& =\sqrt{h_{21}} E\left[X_{2} \mid T\right] E\left[X_{1} \mid T\right]
\end{aligned}
$$


We will now construct another triple $\left(T^{\prime}, X_{1}, X_{2}\right)$ with a covariance matrix $S$ by selecting

$$
T^{\prime}=E\left[X_{1} \mid T\right] .
$$

This particular selection is closely related to the recent work of Bross, Lapidoth and Wigger [18] where it was shown that jointly Gaussian distributions are sufficient to characterize the capacity region of Gaussian MAC with conferencing encoders.

Returning to (161), we note that $T^{\prime}$ is a deterministic function of $T$ and therefore, following is a valid Markov chain.

$$
T^{\prime} \rightarrow T \rightarrow\left(X_{1}, X_{2}\right) \rightarrow\left(Y, Y_{F_{1}}, Y_{F_{2}}\right) .
$$

We will now obtain the off diagonal elements of the covariance matrix $S$ of the triple $\left(T^{\prime}, X_{1}, X_{2}\right)$ as follows:

$$
\begin{aligned}
E\left[X_{1} T^{\prime}\right] & =E_{T}\left[E\left[X_{1} T^{\prime} \mid T\right]\right] \\
& =E_{T}\left[E\left[X_{1} \mid T\right] E\left[X_{1} \mid T\right]\right] \\
& =\operatorname{Var}\left(T^{\prime}\right)
\end{aligned}
$$

and

$$
\begin{aligned}
E\left[X_{2} T^{\prime}\right] & =E_{T}\left[E\left[X_{2} T^{\prime} \mid T\right]\right] \\
& =E_{T}\left[E\left[X_{2} \mid T\right] E\left[X_{1} \mid T\right]\right]
\end{aligned}
$$

and finally:

$$
\begin{aligned}
E\left[X_{1} X_{2}\right] & =E_{T}\left[E\left[X_{1} X_{2} \mid T\right]\right] \\
& =E_{T}\left[E\left[X_{1} \mid T\right] E\left[X_{2} \mid T\right]\right]
\end{aligned}
$$

where (169) follows from (160). Therefore, the triple $\left(T^{\prime}, X_{1}, X_{2}\right)$ satisfies

$$
E\left[X_{1} X_{2}\right]=\frac{E\left[X_{1} T^{\prime}\right] E\left[X_{2} T^{\prime}\right]}{\operatorname{Var}\left(T^{\prime}\right)}
$$

Now using the fact that

$$
\begin{aligned}
E\left[X_{1} X_{2}\right] & =\rho_{12} \sqrt{P_{1} P_{2}} \\
E\left[X_{1} T^{\prime}\right] & =\rho_{1 T^{\prime}} \sqrt{P_{1} P_{T^{\prime}}} \\
E\left[X_{2} T^{\prime}\right] & =\rho_{2 T^{\prime}} \sqrt{P_{2} P_{T^{\prime}}}
\end{aligned}
$$

and substituting in (170) we obtain that the covariance matrix $S$ satisfies

$$
\rho_{12}=\rho_{1 T^{\prime}} \rho_{2 T^{\prime}}
$$

Therefore, from (152) any jointly Gaussian $\left(T_{G}^{\prime}, X_{1 G}, X_{2 G}\right)$ triple with a covariance matrix $S$, with entries $\left(\rho_{12}, \rho_{1 T^{\prime}}, \rho_{2 T^{\prime}}\right)$ satisfies (43).

We now arrive at the final step of the evaluation. In particular, we will show that the rates of this jointly Gaussian triple $\left(T_{G}^{\prime}, X_{1 G}, X_{2 G}\right)$ will include the rates of the given non-Gaussian triple $\left(T, X_{1}, X_{2}\right)$. For the triple $\left(T_{G}^{\prime}, X_{1 G}, X_{2 G}\right)$, we have the following set of inequalities

$$
\begin{aligned}
I\left(X_{1 G}\right. & \left.; Y, Y_{F_{2}} \mid X_{2 G}, T_{G}^{\prime}\right) \\
= & h\left(Y, Y_{F_{2}} \mid X_{2 G}, T_{G}^{\prime}\right)-h\left(Y, Y_{F_{2}} \mid X_{1 G}, X_{2 G}, T_{G}^{\prime}\right) \\
= & h\left(\sqrt{h_{10}} X_{1 G}+Z, \sqrt{h_{12}} X_{1 G}+Z_{2} \mid X_{2 G}, T_{G}^{\prime}\right) \\
& -h\left(Y, Y_{F_{2}} \mid X_{1 G}, X_{2 G}, T_{G}^{\prime}\right) \\
\geq & h\left(\sqrt{h_{10}} X_{1}+Z, \sqrt{h_{12}} X_{1}+Z_{2} \mid X_{2}, T^{\prime}\right) \\
& -h\left(Y, Y_{F_{2}} \mid X_{1 G}, X_{2 G}, T_{G}^{\prime}\right) \\
\geq & h\left(\sqrt{h_{10}} X_{1}+Z, \sqrt{h_{12}} X_{1}+Z_{2} \mid X_{2}, T^{\prime}, T\right) \\
& -h\left(Y, Y_{F_{2}} \mid X_{1}, X_{2}, T\right)
\end{aligned}
$$

$$
\begin{aligned}
= & h\left(\sqrt{h_{10}} X_{1}+Z, \sqrt{h_{12}} X_{1}+Z_{2} \mid X_{2}, T\right) \\
& -h\left(Y, Y_{F_{2}} \mid X_{1}, X_{2}, T\right) \\
= & I\left(X_{1} ; Y, Y_{F_{2}} \mid X_{2}, T\right)
\end{aligned}
$$

where (177) follows from the fact that $\left(T^{\prime}, X_{1}, X_{2}\right)$ and $\left(T_{G}^{\prime}, X_{1 G}, X_{2 G}\right)$ have the same covariance matrix $S$ and by using the maximum entropy theorem. Next, (178) follows from the fact that conditioning reduces differential entropy and finally (179) follows from the fact that $T^{\prime}$ is a deterministic function of $T$ and by invoking the Markov chain in (162). Similarly, we also have

$$
I\left(X_{2 G} ; Y, Y_{F_{1}} \mid X_{1 G}, T_{G}^{\prime}\right) \geq I\left(X_{2} ; Y, Y_{F_{1}} \mid X_{1}, T\right)
$$

$I\left(X_{1 G}, X_{2 G} ; Y, Y_{F_{1}}, Y_{F_{2}} \mid T_{G}^{\prime}\right) \geq I\left(X_{1}, X_{2} ; Y, Y_{F_{1}}, Y_{F_{2}} \mid T\right)$.

Finally, we have

$$
\begin{aligned}
I\left(X_{1 G}, X_{2 G} ; Y\right) & =h(Y)-h\left(Y \mid X_{1 G}, X_{2 G}\right) \\
& =h\left(\sqrt{h_{10}} X_{1 G}+\sqrt{h_{20}} X_{2 G}+Z\right)-h(Z) \\
& \geq h\left(\sqrt{h_{10}} X_{1}+\sqrt{h_{20}} X_{2}+Z\right)-h(Z) \\
& =I\left(X_{1}, X_{2} ; Y\right) .
\end{aligned}
$$

Therefore, we conclude that for any non-Gaussian distribution $p\left(t, x_{1}, x_{2}\right) \in \mathcal{P}_{N G}^{D B(b)}$, there exists a jointly Gaussian distribution $p\left(t, x_{1}, x_{2}\right) \in \mathcal{P}_{G}^{D B}$ which satisfies the dependence balance bound (43) and yields a set of rates which include the set of rates given by the fixed non-Gaussian distribution. Hence, it suffices to consider jointly Gaussian distributions in $\mathcal{P}_{G}^{D B}$ to evaluate our outer bound.

The dependence balance based outer bound can now be written in an explicit form as follows:

$$
\begin{gathered}
\mathcal{D B}_{U C}^{M A C}=\bigcup_{\left(\rho_{1 T}, \rho_{2 T}\right) \in[0,1] \times[0,1]}\left\{\left(R_{1}, R_{2}\right):\right. \\
R_{1} \leq \frac{1}{2} \log \left(1+f_{1}\left(\rho_{1 T}\right)\right) \\
R_{2} \leq \frac{1}{2} \log \left(1+f_{2}\left(\rho_{2 T}\right)\right) \\
R_{1}+R_{2} \leq \frac{1}{2} \log \left(1+f_{3}\left(\rho_{1 T}, \rho_{2 T}\right)\right) \\
\left.R_{1}+R_{2} \leq \frac{1}{2} \log \left(1+f_{4}\left(\rho_{1 T}, \rho_{2 T}\right)\right)\right\}
\end{gathered}
$$

where

$$
\begin{aligned}
& f_{1}\left(\rho_{1 T}\right)=\left(1-\rho_{1 T}^{2}\right) P_{1}\left(\frac{h_{10}}{\sigma_{Z}^{2}}+\frac{h_{12}}{\sigma_{Z_{2}}^{2}}\right) \\
& f_{2}\left(\rho_{2 T}\right)=\left(1-\rho_{2 T}^{2}\right) P_{2}\left(\frac{h_{20}}{\sigma_{Z}^{2}}+\frac{h_{21}}{\sigma_{Z_{1}}^{2}}\right)
\end{aligned}
$$

$$
\text { and }
$$

$$
\begin{aligned}
f_{3}\left(\rho_{1 T}, \rho_{2 T}\right)= & f_{1}\left(\rho_{1 T}\right)+f_{2}\left(\rho_{2 T}\right) \\
& +\left(1-\rho_{1 T}^{2}\right)\left(1-\rho_{2 T}^{2}\right) P_{1} P_{2} \beta
\end{aligned}
$$

$$
f_{4}\left(\rho_{1 T}, \rho_{2 T}\right)=\frac{\left(h_{10} P_{1}+h_{20} P_{2}+2 \rho_{1 T} \rho_{2 T} \sqrt{h_{10} h_{20} P_{1} P_{2}}\right)}{\sigma_{Z}^{2}}
$$

where

$$
\beta=\frac{\left(h_{12} h_{21} \sigma_{Z}^{2}+h_{20} h_{12} \sigma_{Z_{1}}^{2}+h_{10} h_{21} \sigma_{Z_{2}}^{2}\right)}{\sigma_{Z}^{2} \sigma_{Z_{1}}^{2} \sigma_{Z_{2}}^{2}} .
$$


The cut-set outer bound given in (1)-(4) is evaluated for the Gaussian MAC with user cooperation described in (34)-(36) as (193), shown at the bottom of the page.

We now mention how our outer bound compares with the cut-set bound for the limiting cases of cooperation noise variances.

1) $\sigma_{Z_{1}}^{2}, \sigma_{Z_{2}}^{2} \rightarrow 0$ : this case corresponds to total cooperation between transmitters. In this case, both dependence balance bound and the cut-set bound degenerate to the total cooperation line,

$R_{1}+R_{2} \leq \frac{1}{2} \log \left(1+\frac{h_{10} P_{1}+h_{20} P_{2}+2 \sqrt{h_{10} h_{20} P_{1} P_{2}}}{\sigma_{Z}^{2}}\right)$.

2) $\sigma_{Z_{1}}^{2}, \sigma_{Z_{2}}^{2} \rightarrow \infty$ : this case corresponds to very noisy cooperation links. In this case, we have

$$
\begin{aligned}
& f_{1}\left(\rho_{1 T}\right)=\frac{\left(1-\rho_{1 T}^{2}\right) h_{10} P_{1}}{\sigma_{Z}^{2}} \\
& f_{2}\left(\rho_{2 T}\right)=\frac{\left(1-\rho_{2 T}^{2}\right) h_{20} P_{2}}{\sigma_{Z}^{2}} \\
& \text { and } \\
& f_{3}\left(\rho_{1 T}, \rho_{2 T}\right) \\
& \quad=f_{1}\left(\rho_{1 T}\right)+f_{2}\left(\rho_{2 T}\right) \\
& \quad<\frac{\left(h_{10} P_{1}+h_{20} P_{2}+2 \rho_{1 T} \rho_{2 T} \sqrt{h_{10} h_{20} P_{1} P_{2}}\right)}{\sigma_{Z}^{2}}
\end{aligned}
$$

and the dependence balance bound collapses to the capacity region of the Gaussian MAC with no cooperation. On the other hand, the cut-set bound collapses to the capacity region of the Gaussian MAC with noiseless feedback [10].

Fig. 11 illustrates the outer bounds and achievable rate region [14] for the case when $P_{1}=P_{2}=5, \sigma_{Z}^{2}=2$ and $\sigma_{Z_{1}}^{2}=\sigma_{Z_{2}}^{2}=$ 1 and $h_{10}=h_{20}=h_{12}=h_{21}=1$. Fig. 12 illustrates the outer bounds for the case when $P_{1}=P_{2}=\sigma_{Z}^{2}=1$ and $\sigma_{Z_{1}}^{2}=$ $\sigma_{Z_{2}}^{2}=20$ and $h_{10}=h_{20}=h_{12}=h_{21}=1$. For this case, the achievable rate region does not provide any visual improvement over no-cooperation. Fig. 13 illustrates these bounds and the achievable rate region for the asymmetric setting where $P_{1}=$ $P_{2}=\sigma_{Z}^{2}=1$ and $\sigma_{Z_{1}}^{2}=\sigma_{Z_{2}}^{2}=1$ and $h_{10}=h_{20}=1$, $h_{12}=3, h_{21}=2$. Fig. 14 illustrates these bounds and the achievable rate region for the one sided cooperation where $P_{1}=$ $P_{2}=\sigma_{Z}^{2}=1$ and $\sigma_{Z_{1}}^{2}=\sigma_{Z_{2}}^{2}=1$ and $h_{10}=h_{20}=1$, $h_{12}=2, h_{21}=0$.

\section{Evaluation of $\mathcal{D} \mathcal{B}_{U C}^{I C}$}

In this section, we will explicitly evaluate Theorem 5 for the Gaussian IC with user cooperation described by (44)-(47) in Section VIII. We start with step 1 and first characterize the set of jointly Gaussian triples $\left(T_{G}, X_{1 G}, X_{2 G}\right)$ in $\mathcal{P}_{G}^{D B}$. For this purpose, we rewrite (56) as follows:

$$
\begin{aligned}
& h\left(Y_{F_{1}}, Y_{F_{2}} \mid T\right)+h\left(Y_{F_{1}}, Y_{F_{2}} \mid X_{1}, X_{2}, T\right) \\
& \quad \leq h\left(Y_{F_{1}}, Y_{F_{2}} \mid X_{1}, T\right)+h\left(Y_{F_{1}}, Y_{F_{2}} \mid X_{2}, T\right)
\end{aligned}
$$

which can be simplified as in the derivation of $\mathcal{D} \mathcal{B}_{U C}^{M A C}$ to the following two equalities:

$$
\begin{aligned}
I\left(Y_{F_{1}} ; X_{1} \mid T\right) & =0 \\
I\left(Y_{F_{2}} ; X_{2} \mid Y_{F_{1}}, T\right) & =0 .
\end{aligned}
$$

We next follow the same set of arguments used in Section XI to arrive at the fact that a jointly Gaussian triple $\left(T, X_{1}, X_{2}\right)$ satisfies (200)-(201) iff $X_{1} \rightarrow T \rightarrow X_{2}$.

We can now write the set of rate pairs provided by our outer bound for a jointly Gaussian triple $\left(T_{G}, X_{1 G}, X_{2 G}\right)$ in the set $\mathcal{P}_{G}^{D B}$ as

$$
\begin{aligned}
R_{1} & \leq I\left(X_{1 G}, X_{2 G} ; Y_{1}\right) \\
& R_{2} \leq I\left(X_{1 G}, X_{2 G} ; Y_{2}\right) \\
& R_{1} \leq I\left(X_{1 G} ; Y_{1}, Y_{F_{2}} \mid X_{2 G}, T\right) \\
R_{2} & \leq I\left(X_{2 G} ; Y_{2}, Y_{F_{1}} \mid X_{1 G}, T\right) \\
R_{1}+R_{2} & \leq I\left(X_{1 G}, X_{2 G} ; Y_{1}, Y_{2}, Y_{F_{1}}, Y_{F_{2}} \mid T\right) \\
R_{1}+R_{2} & \left.\leq I\left(X_{1 G}, X_{2 G} ; Y_{1}, Y_{2}\right)\right\}
\end{aligned}
$$

where the triple $\left(T_{G}, X_{1 G}, X_{2 G}\right)$ satisfies the Markov chain $X_{1 G} \rightarrow T_{G} \rightarrow X_{2 G}$. Moreover, from the evaluation of step 2 in Section IX, we know that all rate pairs contributed by input distributions in $\mathcal{P}_{N G}^{D B(a)}$ are covered by those given in $\mathcal{P}_{G}^{D B}$. Therefore, we do not need to consider the set $\mathcal{P}_{N G}^{D B(a)}$ in evaluating our outer bound.

We now arrive at step 3 of the evaluation of our outer bound for the Gaussian IC with user cooperation. Consider any triple $\left(T, X_{1}, X_{2}\right)$ with a non-Gaussian distribution $p\left(t, x_{1}, x_{2}\right) \in$ $\mathcal{P}_{N G}^{D B(b)}$, with a valid covariance matrix $Q$. As in the derivation

$$
\begin{aligned}
\mathcal{C S}_{U C}^{M A C} & =\bigcup_{\rho \in[0,1]}\left\{\left(R_{1}, R_{2}\right):\right. \\
R_{1} & \leq \frac{1}{2} \log \left(1+\left(1-\rho^{2}\right) P_{1}\left(\frac{h_{10}}{\sigma_{Z}^{2}}+\frac{h_{12}}{\sigma_{Z_{2}}^{2}}\right)\right) \\
R_{2} & \leq \frac{1}{2} \log \left(1+\left(1-\rho^{2}\right) P_{2}\left(\frac{h_{20}}{\sigma_{Z}^{2}}+\frac{h_{21}}{\sigma_{Z_{1}}^{2}}\right)\right) \\
R_{1}+R_{2} & \left.\leq \frac{1}{2} \log \left(1+\frac{h_{10} P_{1}+h_{20} P_{2}+2 \rho \sqrt{h_{10} h_{20} P_{1} P_{2}}}{\sigma_{Z}^{2}}\right)\right\}
\end{aligned}
$$




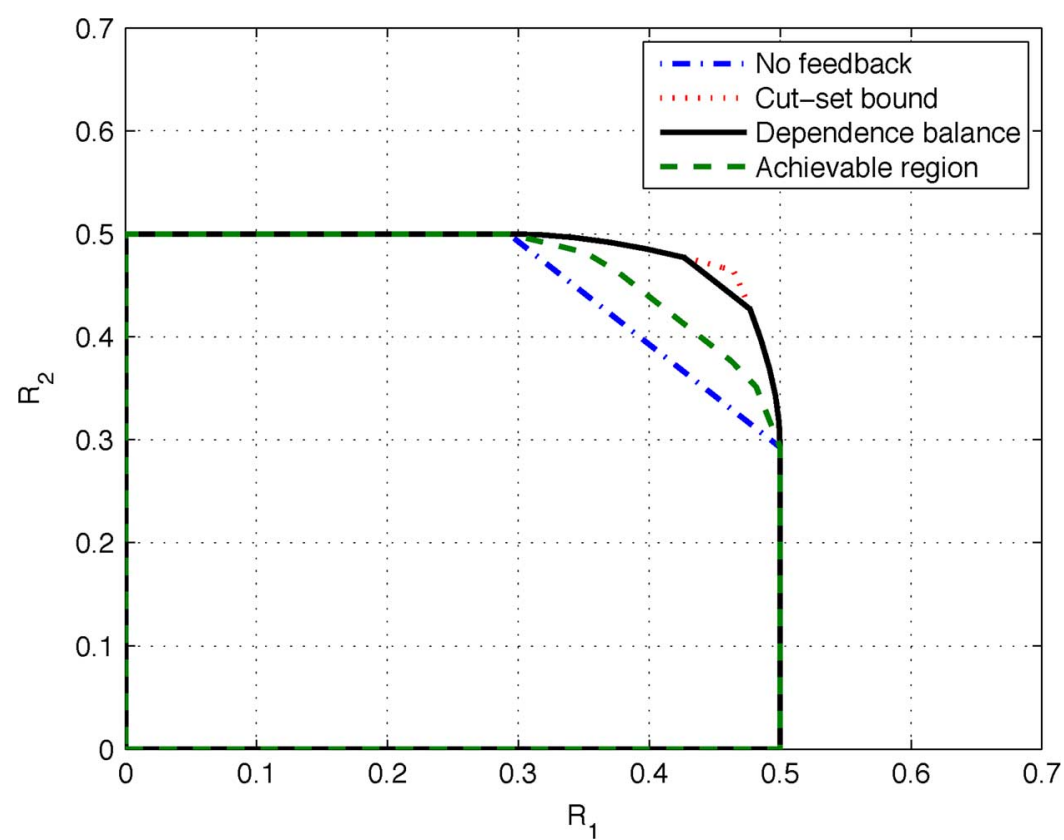

Fig. 9. Illustration of outer bound and an achievable region based on superposition coding for $P_{1}=P_{2}=\sigma_{Z}^{2}=1$ and $\sigma_{Z_{1}}^{2}=\sigma_{Z_{2}}^{2}=0.3$.

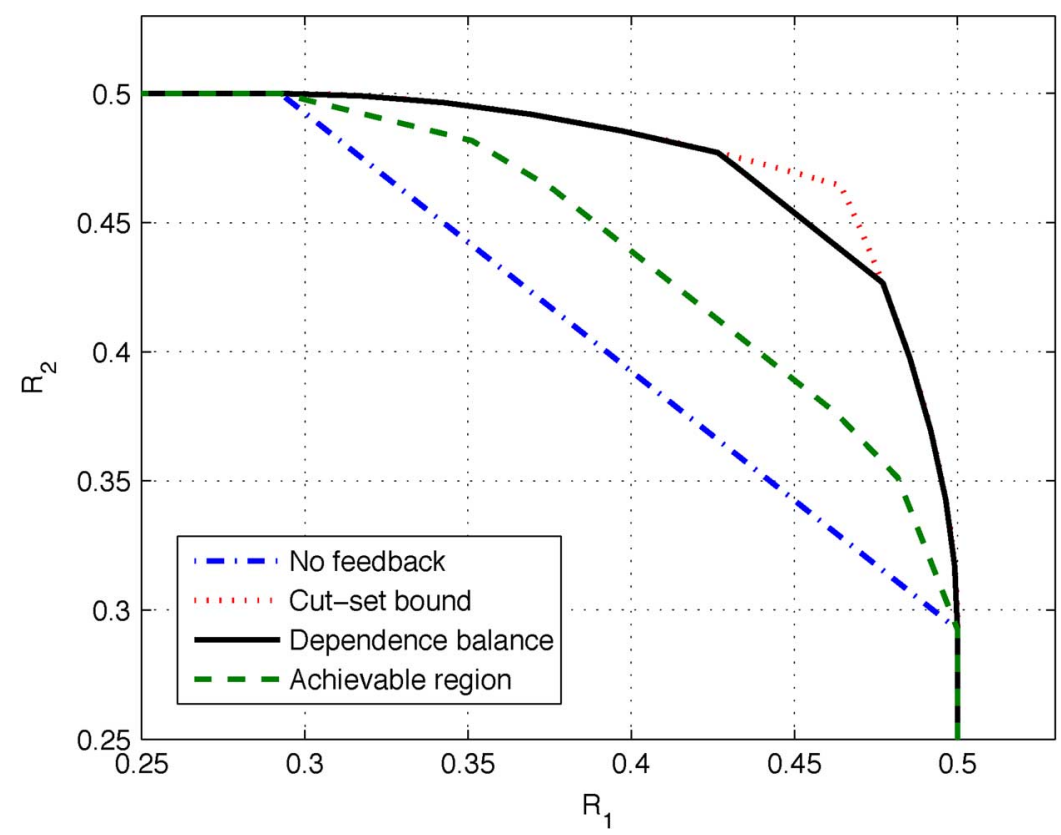

Fig. 10. Enlarged illustration of Fig. 9.

of $\mathcal{D} \mathcal{B}_{U C}^{M A C}$, we first construct another triple $\left(T^{\prime}, X_{1}, X_{2}\right)$ with a covariance matrix $S$ by selecting

$$
T^{\prime}=E\left[X_{1} \mid T\right]
$$

Following this step, we next make use of the Markov chain

$$
T^{\prime} \rightarrow T \rightarrow\left(X_{1}, X_{2}\right) \rightarrow\left(Y_{1}, Y_{2}, Y_{F_{1}}, Y_{F_{2}}\right)
$$

to show the existence of a jointly Gaussian $\left(T_{G}^{\prime}, X_{1 G}, X_{2 G}\right)$ with a covariance matrix $S$ and which satisfies (56).

We now arrive at the final step of the evaluation. In particular, we will show that the rates of this jointly Gaussian triple $\left(T_{G}^{\prime}, X_{1 G}, X_{2 G}\right)$ will include the rates of the given non-Gaussian triple $\left(T, X_{1}, X_{2}\right)$. For the triple $\left(T_{G}^{\prime}, X_{1 G}, X_{2 G}\right)$, we have the following set of inequalities:

$$
\begin{aligned}
& I\left(X_{1 G} ; Y_{1}, Y_{F_{2}} \mid X_{2 G}, T_{G}^{\prime}\right) \\
&=h\left(Y_{1}, Y_{F_{2}} \mid X_{2 G}, T_{G}^{\prime}\right)-h\left(Y_{1}, Y_{F_{2}} \mid X_{1 G}, X_{2 G}, T_{G}^{\prime}\right) \\
&=h\left(X_{1 G}+N_{1}, \sqrt{h_{12}} X_{1 G}+Z_{2} \mid X_{2 G}, T_{G}^{\prime}\right) \\
& \quad-h\left(Y_{1}, Y_{F_{2}} \mid X_{1 G}, X_{2 G}, T_{G}^{\prime}\right) \\
& \geq h\left(X_{1}+N_{1}, \sqrt{h_{12}} X_{1}+Z_{2} \mid X_{2}, T^{\prime}\right) \\
&-h\left(Y_{1}, Y_{F_{2}} \mid X_{1 G}, X_{2 G}, T_{G}^{\prime}\right)
\end{aligned}
$$




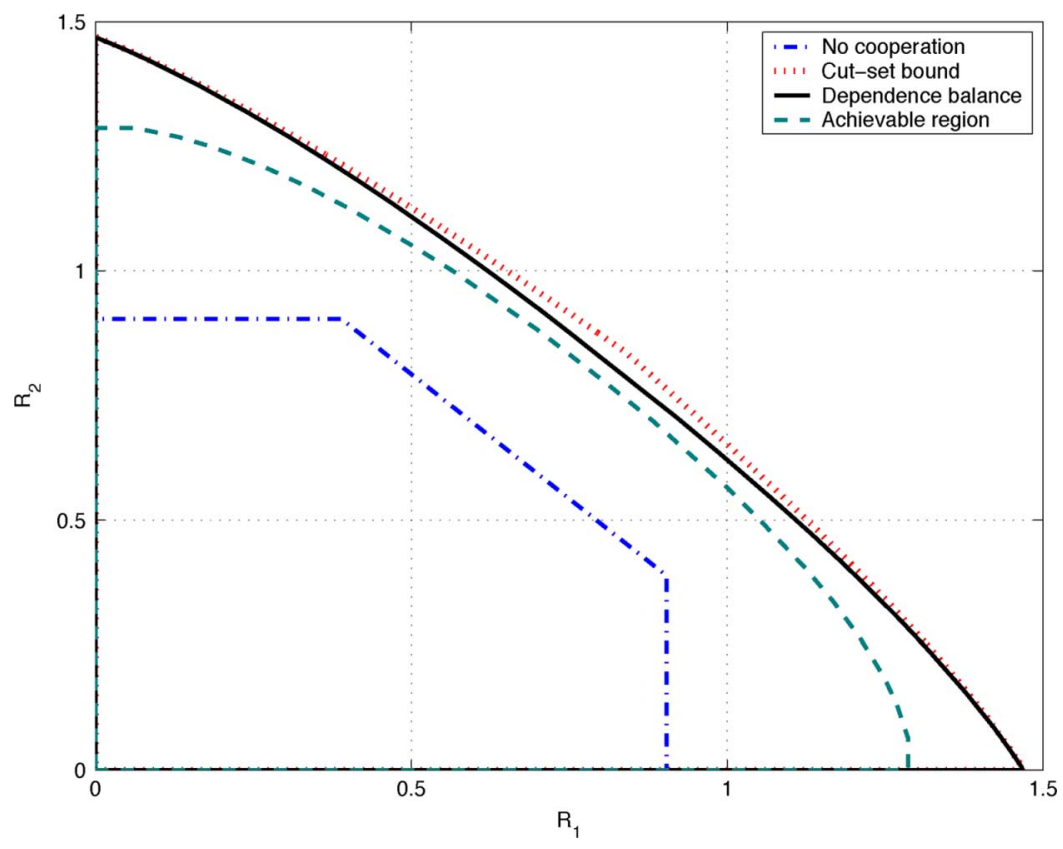

Fig. 11. Illustration of bounds for $P_{1}=P_{2}=5, \sigma_{Z}^{2}=2, \sigma_{Z_{1}}^{2}=\sigma_{Z_{2}}^{2}=1$ and $h_{10}=h_{20}=h_{12}=h_{21}=1$.

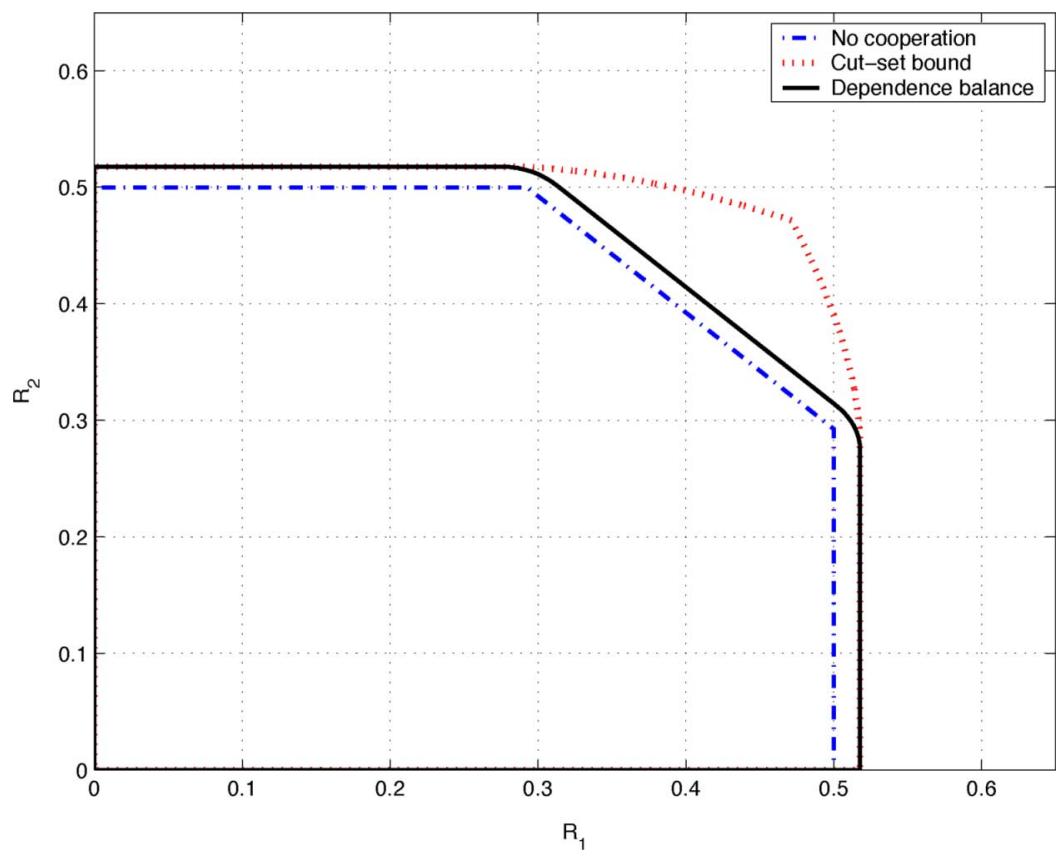

Fig. 12. Illustration of outer bounds for $P_{1}=P_{2}=\sigma_{Z}^{2}=1, \sigma_{Z_{1}}^{2}=\sigma_{Z_{2}}^{2}=20$ and $h_{10}=h_{20}=h_{12}=h_{21}=1$.

$$
\begin{aligned}
\geq & h\left(X_{1}+N_{1}, \sqrt{h_{12}} X_{1}+Z_{2} \mid X_{2}, T^{\prime}, T\right) \\
& -h\left(Y_{1}, Y_{F_{2}} \mid X_{1 G}, X_{2 G}, T_{G}^{\prime}\right) \\
= & h\left(X_{1}+N_{1}, \sqrt{h_{12}} X_{1}+Z_{2} \mid X_{2}, T\right) \\
& -h\left(Y_{1}, Y_{F_{2}} \mid X_{1}, X_{2}, T\right) \\
= & I\left(X_{1} ; Y_{1}, Y_{F_{2}} \mid X_{2}, T\right)
\end{aligned}
$$

where (212) follows from the fact that $\left(T^{\prime}, X_{1}, X_{2}\right)$ and $\left(T_{G}^{\prime}, X_{1 G}, X_{2 G}\right)$ have the same covariance matrix $S$ and using the maximum entropy theorem. Next, (213) follows from the fact that conditioning reduces differential entropy and finally
(214) follows from the fact that $T^{\prime}$ is a deterministic function of $T$ and invoking the Markov chain in (209). Similarly, we also have

$$
I\left(X_{2 G} ; Y_{2}, Y_{F_{1}} \mid X_{1 G}, T_{G}^{\prime}\right) \geq I\left(X_{2} ; Y_{2}, Y_{F_{1}} \mid X_{1}, T\right)
$$

and

$$
\begin{aligned}
& I\left(X_{1 G}, X_{2 G} ; Y_{1}, Y_{2}, Y_{F_{1}}, Y_{F_{2}} \mid T_{G}^{\prime}\right) \\
& \quad \geq I\left(X_{1}, X_{2} ; Y_{1}, Y_{2}, Y_{F_{1}}, Y_{F_{2}} \mid T\right) .
\end{aligned}
$$




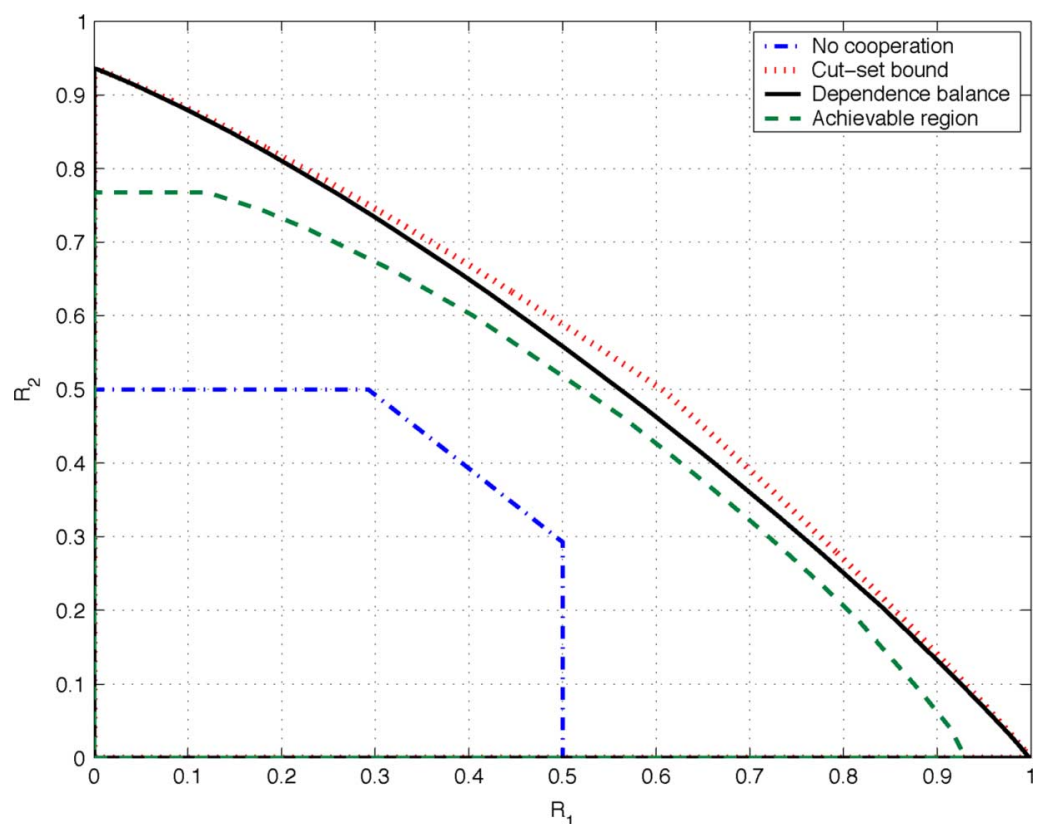

Fig. 13. Illustration of outer bounds for $P_{1}=P_{2}=\sigma_{Z}^{2}=\sigma_{Z_{1}}^{2}=\sigma_{Z_{2}}^{2}=1$ and $h_{10}=h_{20}=1, h_{12}=3, h_{21}=2$.

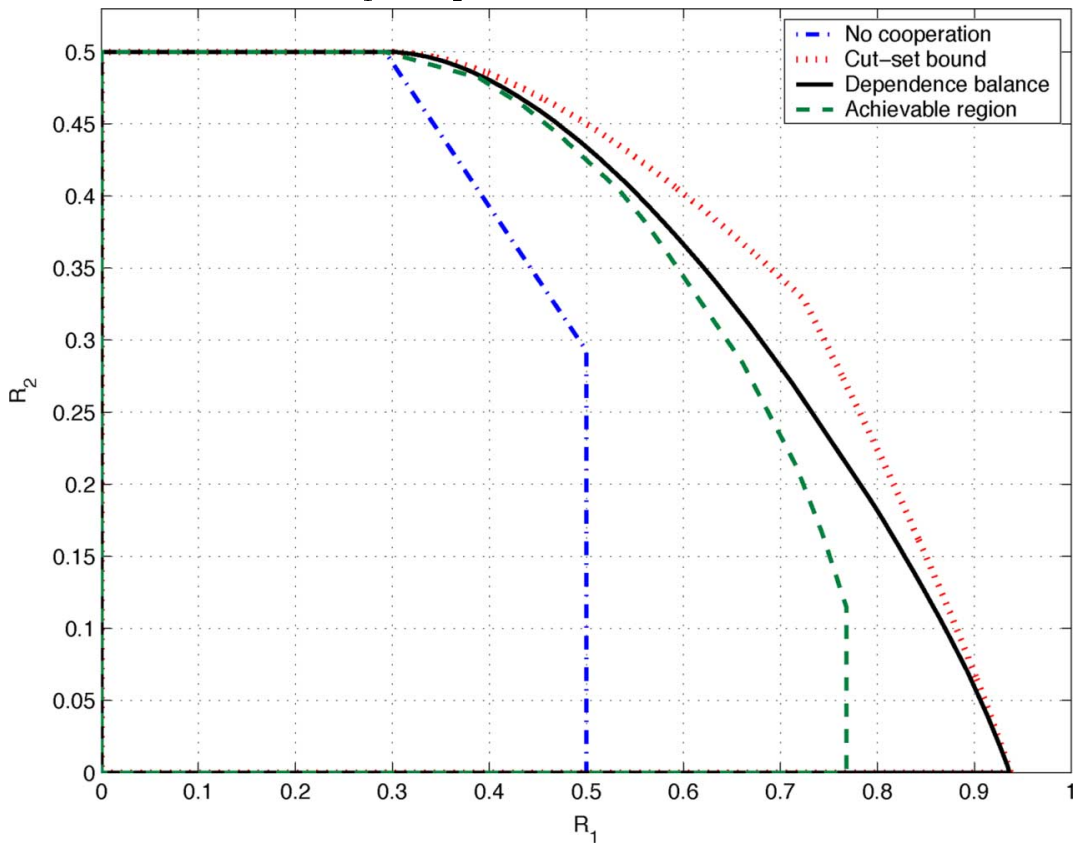

Fig. 14. Illustration of outer bounds for $P_{1}=P_{2}=\sigma_{Z}^{2}=\sigma_{Z_{1}}^{2}=\sigma_{Z_{2}}^{2}=1$ and $h_{10}=h_{20}=1, h_{12}=2, h_{21}=0$.

As in the derivation of $\mathcal{D} \mathcal{B}_{U C}^{M A C}$, we also have

$$
\begin{gathered}
I\left(X_{1 G}, X_{2 G} ; Y_{1}\right) \geq I\left(X_{1}, X_{2} ; Y_{1}\right) \\
I\left(X_{1 G}, X_{2 G} ; Y_{2}\right) \geq I\left(X_{1}, X_{2} ; Y_{2}\right) \\
I\left(X_{1 G}, X_{2 G} ; Y_{1}, Y_{2}\right) \geq I\left(X_{1}, X_{2} ; Y_{1}, Y_{2}\right) .
\end{gathered}
$$

Therefore, we conclude that for any non-Gaussian distribution $p\left(t, x_{1}, x_{2}\right) \in \mathcal{P}_{N G}^{D B(b)}$, there exists a jointly Gaussian distribution $p\left(t, x_{1}, x_{2}\right) \in \mathcal{P}_{G}^{D B}$ which satisfies the dependence balance bound (56) and yields a set of rates which includes the set of rates given by the fixed non-Gaussian distribution. Hence, it suffices to consider jointly Gaussian distributions in $\mathcal{P}_{G}^{D B}$ to evaluate our outer bound.
The dependence balance based outer bound can now be written in an explicit form as

$$
\begin{aligned}
\mathcal{D} \mathcal{B}_{U C}^{I C}=\left(\rho_{1 T}, \rho_{2 T}\right) \in[0,1] \times[0,1]\left\{\left(R_{1}, R_{2}\right):\right. \\
R_{1} \leq \frac{1}{2} \log \left(1+f_{1}\left(\rho_{1 T}, \rho_{2 T}\right)\right) \\
R_{2} \leq \frac{1}{2} \log \left(1+f_{2}\left(\rho_{1 T}, \rho_{2 T}\right)\right) \\
R_{1} \leq \frac{1}{2} \log \left(1+f_{3}\left(\rho_{1 T}\right)\right) \\
R_{2} \leq \frac{1}{2} \log \left(1+f_{4}\left(\rho_{2 T}\right)\right) \\
R_{1}+R_{2} \leq \frac{1}{2} \log \left(1+f_{5}\left(\rho_{1 T}, \rho_{2 T}\right)\right) \\
\left.R_{1}+R_{2} \leq \frac{1}{2} \log \left(1+f_{6}\left(\rho_{1 T}, \rho_{2 T}\right)\right)\right\}
\end{aligned}
$$




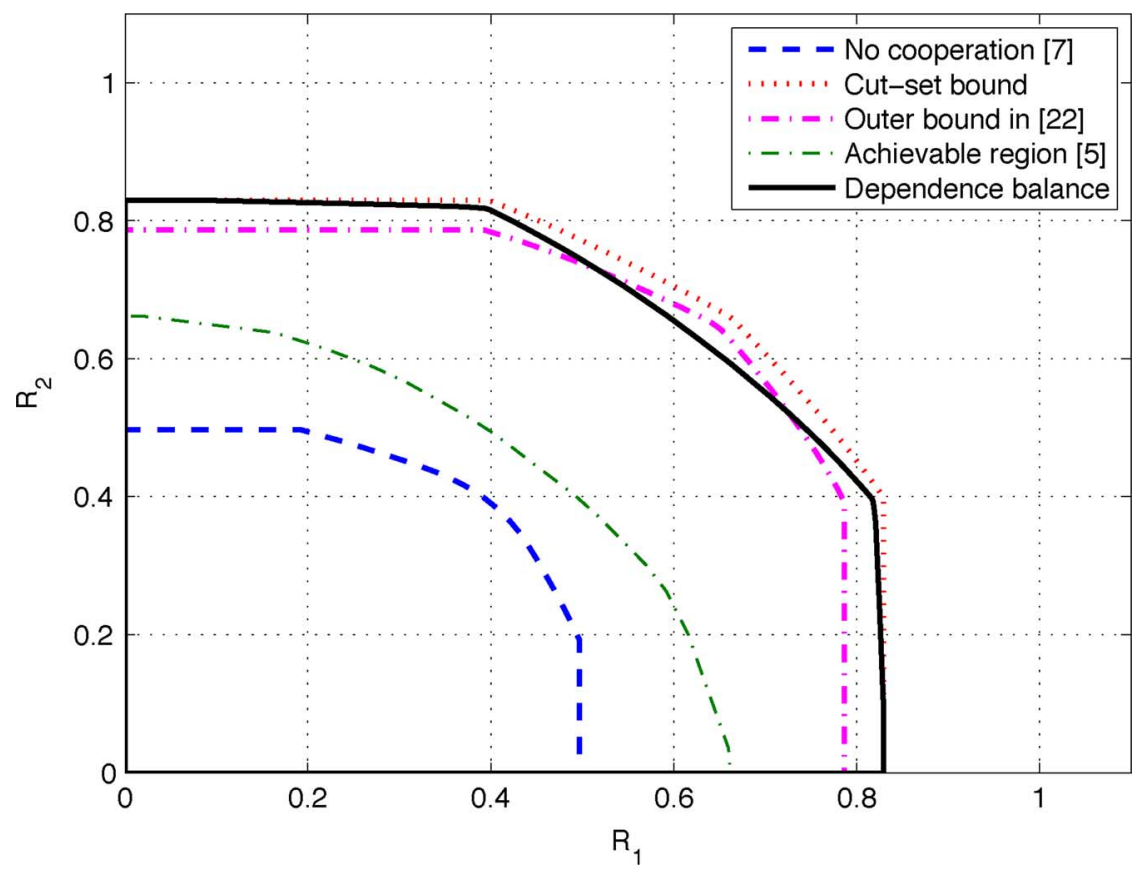

Fig. 15. Illustration of bounds for $P_{1}=P_{2}=\sigma_{N_{1}}^{2}=\sigma_{N_{2}}^{2}=1, \sigma_{Z_{1}}^{2}=\sigma_{Z_{2}}^{2}=1$ and $a=b=0.6$ and $h_{12}=h_{21}=1.5$.

where

$$
\begin{aligned}
f_{1}\left(\rho_{1 T}, \rho_{2 T}\right)= & \frac{\left(P_{1}+b P_{2}+2 \rho_{1 T} \rho_{2 T} \sqrt{b P_{1} P_{2}}\right)}{\sigma_{N_{1}}^{2}} \\
f_{2}\left(\rho_{1 T}, \rho_{2 T}\right)= & \frac{\left(a P_{1}+P_{2}+2 \rho_{1 T} \rho_{2 T} \sqrt{a P_{1} P_{2}}\right)}{\sigma_{N_{2}}^{2}} \\
f_{3}\left(\rho_{1 T}\right)= & \left(1-\rho_{1 T}^{2}\right) P_{1}\left(\frac{1}{\sigma_{N_{1}}^{2}}+\frac{h_{12}}{\sigma_{Z_{2}}^{2}}\right) \\
f_{4}\left(\rho_{2 T}\right)= & \left(1-\rho_{2 T}^{2}\right) P_{2}\left(\frac{1}{\sigma_{N_{2}}^{2}}+\frac{h_{21}}{\sigma_{Z_{1}}^{2}}\right) \\
f_{5}\left(\rho_{1 T}, \rho_{2 T}\right)= & f_{1}\left(\rho_{1 T}, \rho_{2 T}\right)+f_{2}\left(\rho_{1 T}, \rho_{2 T}\right) \\
& +\frac{\left(1-\rho_{1 T}^{2} \rho_{2 T}^{2}\right) P_{1} P_{2}(1-\sqrt{a b})^{2}}{\sigma_{N_{1}}^{2} \sigma_{N_{2}}^{2}}
\end{aligned}
$$

$$
\begin{aligned}
f_{6}\left(\rho_{1 T}, \rho_{2 T}\right)= & f_{3}\left(\rho_{1 T}\right)+f_{4}\left(\rho_{2 T}\right) \\
& +\frac{\left(1-\rho_{1 T}^{2}\right) a P_{1}}{\sigma_{N_{2}}^{2}}+\frac{\left(1-\rho_{2 T}^{2}\right) b P_{2}}{\sigma_{N_{1}}^{2}} \\
& +\left(1-\rho_{1 T}^{2}\right)\left(1-\rho_{2 T}^{2}\right) P_{1} P_{2} \beta
\end{aligned}
$$

where

$$
\begin{aligned}
\beta= & \frac{h_{12} h_{21}}{\sigma_{Z_{1}}^{2} \sigma_{Z_{2}}^{2}}+\frac{(1-\sqrt{a b})^{2}}{\sigma_{N_{1}}^{2} \sigma_{N_{2}}^{2}}+\frac{h_{12}}{\sigma_{Z_{2}}^{2}}\left(\frac{1}{\sigma_{N_{2}}^{2}}+\frac{b}{\sigma_{N_{1}}^{2}}\right) \\
& +\frac{h_{21}}{\sigma_{Z_{1}}^{2}}\left(\frac{1}{\sigma_{N_{1}}^{2}}+\frac{a}{\sigma_{N_{2}}^{2}}\right) .
\end{aligned}
$$

The cut-set outer bound given in (5)-(10) is evaluated for the Gaussian IC with user cooperation described in (44)-(47) as

$$
\begin{aligned}
\mathcal{C S}_{U C}^{I C} & =\bigcup_{\rho \in[0,1]}\left\{\left(R_{1}, R_{2}\right):\right. \\
R_{1} & \leq \frac{1}{2} \log \left(1+\frac{P_{1}+b P_{2}+2 \rho \sqrt{b P_{1} P_{2}}}{\sigma_{N_{1}}^{2}}\right)
\end{aligned}
$$

$$
\begin{aligned}
R_{2} & \leq \frac{1}{2} \log \left(1+\frac{a P_{1}+P_{2}+2 \rho \sqrt{a P_{1} P_{2}}}{\sigma_{N_{2}}^{2}}\right) \\
R_{1} & \leq \frac{1}{2} \log \left(1+\left(1-\rho^{2}\right) P_{1}\left(\frac{1}{\sigma_{N_{1}}^{2}}+\frac{h_{12}}{\sigma_{Z_{2}}^{2}}\right)\right) \\
R_{2} & \leq \frac{1}{2} \log \left(1+\left(1-\rho^{2}\right) P_{2}\left(\frac{1}{\sigma_{N_{2}}^{2}}+\frac{h_{21}}{\sigma_{Z_{1}}^{2}}\right)\right) \\
R_{1}+R_{2} \leq & \frac{1}{2} \log \left(1+k_{1}(\rho)+k_{2}(\rho)\right. \\
+ & \left.\left.\frac{\left(1-\rho^{2}\right) P_{1} P_{2}(1-\sqrt{a b})^{2}}{\sigma_{N_{1}}^{2} \sigma_{N_{2}}^{2}}\right)\right\}
\end{aligned}
$$

where

$$
k_{1}(\rho)=\frac{P_{1}+b P_{2}+2 \rho \sqrt{b P_{1} P_{2}}}{\sigma_{N_{1}}^{2}}
$$

and

$$
k_{1}(\rho)=\frac{a P_{1}+P_{2}+2 \rho \sqrt{a P_{1} P_{2}}}{\sigma_{N_{2}}^{2}} .
$$

Fig. 15 illustrates our outer bound, cut-set bound, outer bound in [22], an achievable rate region with cooperation [5] and an achievable rate region without cooperation [7] for the case when $P_{1}=P_{2}=\sigma_{N_{1}}^{2}=\sigma_{N_{1}}^{2}=1$ and $\sigma_{Z_{1}}^{2}=\sigma_{Z_{2}}^{2}=1$ and $a=b=0.6$ and $h_{12}=h_{21}=1.5$. Fig. 16 illustrates our sum-rate upper bound, cut-set bound, upper bound in [22], upper bound in [23] as function of $h$, where $h=h_{12}=h_{21}$ and $P_{1}=P_{2}=\sigma_{N_{1}}^{2}=\sigma_{N_{1}}^{2}=1$ and $\sigma_{Z_{1}}^{2}=\sigma_{Z_{2}}^{2}=1, a=b=0.5$. Fig. 17 illustrates our sum-rate upper bound, cut-set bound, and sum-rate bound in [22] as function of $h$, where $h=h_{12}=h_{21}$ and $P_{1}=P_{2}=\sigma_{N_{1}}^{2}=\sigma_{N_{1}}^{2}=1$ and $\sigma_{Z_{1}}^{2}=\sigma_{Z_{2}}^{2}=1$, $a=b=0.1$. For the case of moderate interference, we observe from Fig. 16 that neither the DB bound nor the upper bound in [22] are better than each other. On the other hand, for the 


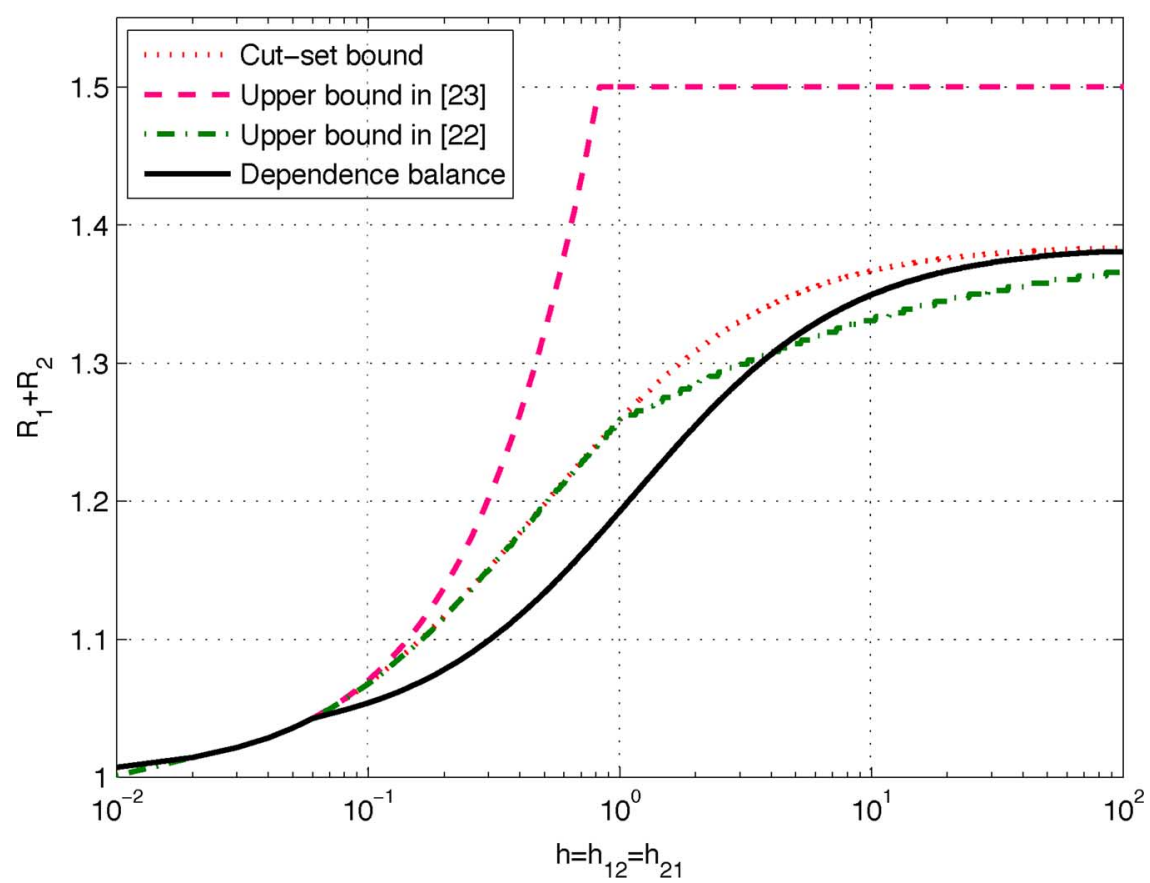

Fig. 16. Illustration of sum-rate upper bounds as functions of $h$, where $h=h_{12}=h_{21}$ and $a=b=0.5$.

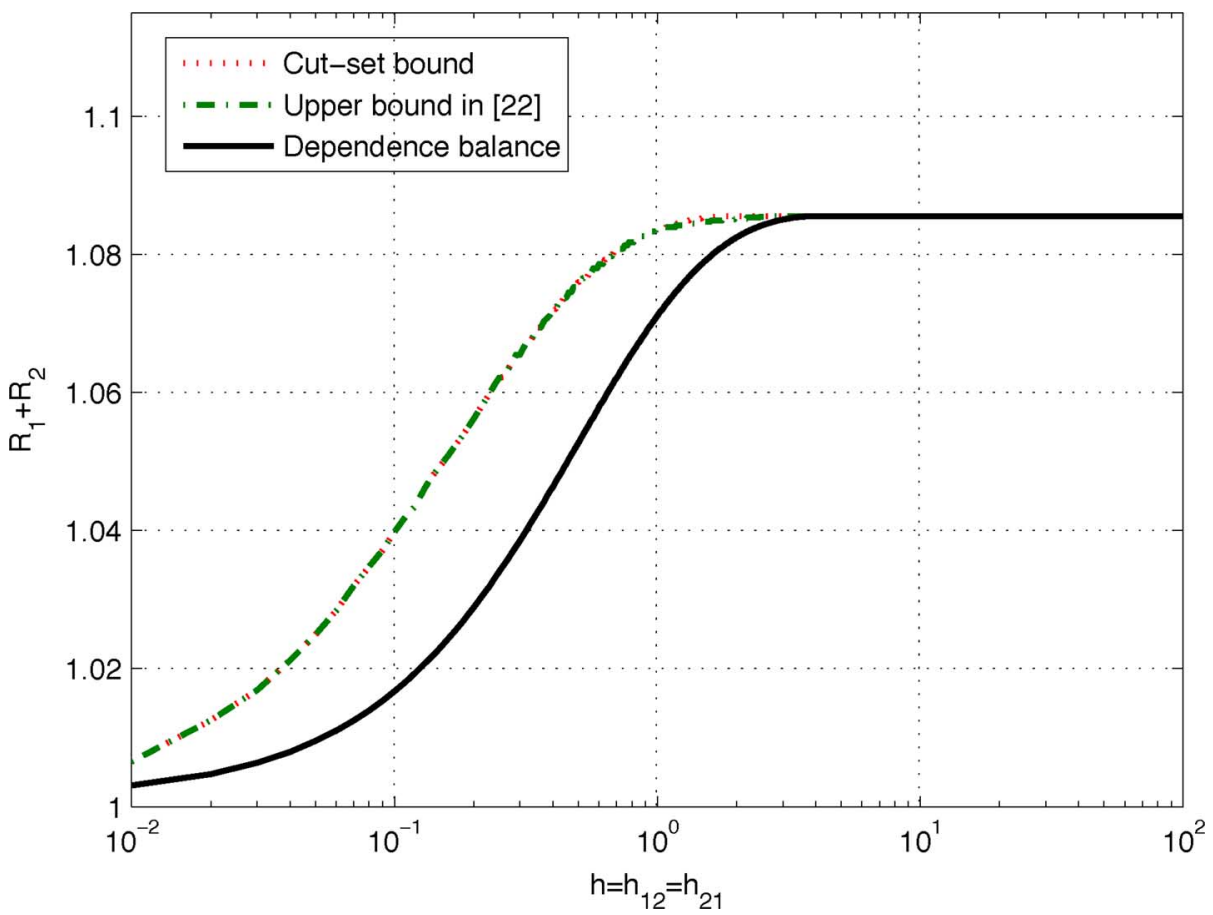

Fig. 17. Illustration of sum-rate upper bounds as functions of $h$, where $h=h_{12}=h_{21}$ and $a=b=0.1$.

case of weak interference, we observe from Fig. 17, that the DB sum-rate bound is strictly less than the upper bound in [22].

\section{CONCLUSION}

We obtained new outer bounds for the capacity regions of the two-user MAC with generalized feedback and the two-user IC with generalized feedback. We explicitly evaluated these outer bounds for three channel models. In particular, we evaluated our outer bounds for the Gaussian MAC with different noisy feedback signals at the transmitters, the Gaussian MAC with user cooperation and the Gaussian IC with user cooperation.
Our outer bounds strictly improve upon the cut-set bound for all three channel models.

For the evaluation of our outer bounds for the Gaussian scenarios of interest, we proposed a systematic approach to deal with capacity bounds involving auxiliary random variables. This approach was appropriately tailored according to the channel model in consideration which permitted us to obtain explicit expressions for our outer bounds. To evaluate our outer bounds, we have to consider all input distributions satisfying the dependence balance constraint. The main difficulty in evaluating our outer bounds arises from the fact that there might exist some 
non-Gaussian input distribution $p\left(t, x_{1}, x_{2}\right)$ with a covariance matrix $Q$, such that $p\left(t, x_{1}, x_{2}\right)$ satisfies the dependence balance constraint but there does not exist a jointly Gaussian triple with the covariance matrix $Q$ satisfying the dependence balance constraint. Therefore, the regular methodology of evaluating outer bounds, i.e., the approach of applying maximum entropy theorem [8] fails beyond this particular point. Through our explicit evaluation for all three channel models, we were able to show the existence of a jointly Gaussian triple with a covariance matrix $S$ which satisfies the dependence balance constraint and yields larger rates than the fixed non-Gaussian distribution.

In particular, for the case of Gaussian MAC with noisy feedback, we made use of a recently discovered multivariate EPI [16], which is a generalization of Costa's EPI [17]. It is worth noting that this result could not be obtained from the classical vector EPI. For the case of Gaussian MAC with user cooperation and the Gaussian IC with user cooperation, our proof closely follows a recent result of Bross, Wigger and Lapidoth [18] and [19] for the Gaussian MAC with conferencing encoders.

\section{APPENDIX I}

\section{A. Proof of Theorem 1}

We will prove Theorem 1 by first deriving an upper bound for $R_{1}$ as (232)-(243), shown at the bottom of the page: where (234) follows from Fano's inequality [8], (237) follows from the fact that $X_{2 i}$ is a function of $\left(W_{2}, Y_{F_{2}}^{i-1}\right)$ and by introducing $X_{2 i}$ in both terms, (238) follows from the fact that conditioning reduces

$$
\begin{aligned}
& n R_{1} \\
& =H\left(W_{1}\right)=H\left(W_{1} \mid W_{2}\right) \\
& =I\left(W_{1} ; Y^{n}, Y_{F_{1}}^{n}, Y_{F_{2}}^{n} \mid W_{2}\right)+H\left(W_{1} \mid W_{2}, Y^{n}, Y_{F_{1}}^{n}, Y_{F_{2}}^{n}\right) \\
& \leq I\left(W_{1} ; Y^{n}, Y_{F_{1}}^{n}, Y_{F_{2}}^{n} \mid W_{2}\right)+n \epsilon_{1}^{(n)} \\
& =\sum_{i=1}^{n} I\left(W_{1} ; Y_{i}, Y_{F_{1} i}, Y_{F_{2} i} \mid W_{2}, Y^{i-1}, Y_{F_{1}}^{i-1}, Y_{F_{2}}^{i-1}\right)+n \epsilon_{1}^{(n)} \\
& =\sum_{i=1}^{n}\left(H\left(Y_{i}, Y_{F_{1} i}, Y_{F_{2} i} \mid W_{2}, Y^{i-1}, Y_{F_{1}}^{i-1}, Y_{F_{2}}^{i-1}\right)\right. \\
& \left.-H\left(Y_{i}, Y_{F_{1} i}, Y_{F_{2} i} \mid W_{1}, W_{2}, Y^{i-1}, Y_{F_{1}}^{i-1}, Y_{F_{2}}^{i-1}\right)\right) \\
& +n \epsilon_{1}^{(n)} \\
& =\sum_{i=1}^{n}\left(H\left(Y_{i}, Y_{F_{1} i}, Y_{F_{2} i} \mid W_{2}, X_{2 i}, Y^{i-1}, Y_{F_{1}}^{i-1}, Y_{F_{2}}^{i-1}\right)\right. \\
& \left.-H\left(Y_{i}, Y_{F_{1} i}, Y_{F_{2} i} \mid X_{2 i}, W_{1}, W_{2}, Y^{i-1}, Y_{F_{1}}^{i-1}, Y_{F_{2}}^{i-1}\right)\right) \\
& +n \epsilon_{1}^{(n)} \\
& \leq \sum_{i=1}^{n}\left(H\left(Y_{i}, Y_{F_{1} i}, Y_{F_{2} i} \mid X_{2 i}, Y_{F_{1}}^{i-1}, Y_{F_{2}}^{i-1}\right)\right. \\
& \left.-H\left(Y_{i}, Y_{F_{1} i}, Y_{F_{2} i} \mid X_{2 i}, W_{1}, W_{2}, Y^{i-1}, Y_{F_{1}}^{i-1}, Y_{F_{2}}^{i-1}\right)\right) \\
& +n \epsilon_{1}^{(n)} \\
& \leq \sum_{i=1}^{n}\left(H\left(Y_{i}, Y_{F_{1} i}, Y_{F_{2} i} \mid X_{2 i}, Y_{F_{1}}^{i-1}, Y_{F_{2}}^{i-1}\right)\right. \\
& \left.-H\left(Y_{i}, Y_{F_{1} i}, Y_{F_{2} i} \mid X_{1 i}, X_{2 i}, W_{1}, W_{2}, Y^{i-1}, Y_{F_{1}}^{i-1}, Y_{F_{2}}^{i-1}\right)\right) \\
& +n \epsilon_{1}^{(n)} \\
& =\sum_{i=1}^{n}\left(H\left(Y_{i}, Y_{F_{1} i}, Y_{F_{2} i} \mid X_{2 i}, Y_{F_{1}}^{i-1}, Y_{F_{2}}^{i-1}\right)\right. \\
& \left.-H\left(Y_{i}, Y_{F_{1} i}, Y_{F_{2} i} \mid X_{1 i}, X_{2 i}, Y_{F_{1}}^{i-1}, Y_{F_{2}}^{i-1}\right)\right)+n \epsilon_{1}^{(n)} \\
& =\sum_{i=1}^{n} I\left(X_{1 i} ; Y_{i}, Y_{F_{1} i}, Y_{F_{2} i} \mid X_{2 i}, Y_{F_{1}}^{i-1}, Y_{F_{2}}^{i-1}\right)+n \epsilon_{1}^{(n)} \\
& =n I\left(X_{1 Q} ; Y_{Q}, Y_{F_{1} Q}, Y_{F_{2} Q} \mid X_{2 Q}, Q, Y_{F_{1}}^{Q-1}, Y_{F_{2}}^{Q-1}\right)+n \epsilon_{1}^{(n)} \\
& =n I\left(X_{1} ; Y, Y_{F_{1}}, Y_{F_{2}} \mid X_{2}, T\right)+n \epsilon_{1}^{(n)}
\end{aligned}
$$


entropy and we drop $\left(W_{2}, Y^{i-1}\right)$ from the conditioning in the first term, (239) follows from the fact that conditioning reduces entropy and by introducing $X_{1 i}$ in the second term and (240) follows from the memoryless property of the channel. Finally, we define $X_{1}=X_{1 Q}, X_{2}=X_{2 Q}, T=\left(Q, Y_{F_{1}}^{Q-1}, Y_{F_{2}}^{Q-1}\right)$, $Y=Y_{Q}, Y_{F_{1}}=Y_{F_{1} Q}$ and $Y_{F_{2}}=Y_{F_{2} Q}$, where $Q$ is a random variable which is uniformly distributed over $\{1, \ldots, n\}$ and is independent of all other random variables. Similarly, we have

$$
\begin{aligned}
R_{2} & \leq I\left(X_{2} ; Y, Y_{F_{1}}, Y_{F_{2}} \mid X_{1}, T\right) \\
R_{1}+R_{2} & \leq I\left(X_{1}, X_{2} ; Y, Y_{F_{1}}, Y_{F_{2}} \mid T\right)
\end{aligned}
$$

In addition to (245), we also have the following sum-rate constraint which also appears in the cut-set outer bound,

$$
R_{1}+R_{2} \leq I\left(X_{1}, X_{2} ; Y\right)
$$

The proof of the dependence balance constraint in (16) is along the same lines as in [9] by starting from the inequality

$$
0 \leq I\left(W_{1} ; W_{2} \mid Y_{F_{1}}^{n}, Y_{F_{2}}^{n}\right)-I\left(W_{1} ; W_{2}\right)
$$

to arrive at

$$
I\left(X_{1} ; X_{2} \mid T\right) \leq I\left(X_{1} ; X_{2} \mid Y_{F_{1}}, Y_{F_{2}}, T\right) .
$$

This completes the Proof of Theorem 1.

\section{B. Proof of Theorem 2}

We will prove Theorem 2 by first deriving an upper bound for $R_{1}$ as (249)-(259), shown at the bottom of the page: where (251) follows from Fano's inequality [8], (254) follows from the fact that $X_{2 i}$ is a function of $\left(W_{2}, Y_{F_{2}}^{i-1}\right)$ and by introducing $X_{2 i}$ in both terms, (255) follows from the fact that conditioning reduces entropy and we drop $\left(W_{2}, Y_{1}^{i-1}\right)$ from the conditioning

$$
\begin{aligned}
& n R_{1} \\
& =H\left(W_{1}\right)=H\left(W_{1} \mid W_{2}\right) \\
& =I\left(W_{1} ; Y_{1}^{n}, Y_{F_{1}}^{n}, Y_{F_{2}}^{n} \mid W_{2}\right)+H\left(W_{1} \mid W_{2}, Y_{1}^{n}, Y_{F_{1}}^{n}, Y_{F_{2}}^{n}\right) \\
& \leq I\left(W_{1} ; Y_{1}^{n}, Y_{F_{1}}^{n}, Y_{F_{2}}^{n} \mid W_{2}\right)+n \epsilon_{1}^{(n)} \\
& =\sum_{i=1}^{n} I\left(W_{1} ; Y_{1 i}, Y_{F_{1}}, Y_{F_{2} i} \mid W_{2}, Y_{1}^{i-1}, Y_{F_{1}}^{i-1}, Y_{F_{2}}^{i-1}\right)+n \epsilon_{1}^{(n)} \\
& =\sum_{i=1}^{n}\left(H\left(Y_{1 i}, Y_{F_{1} i}, Y_{F_{2}} \mid W_{2}, Y_{1}^{i-1}, Y_{F_{1}}^{i-1}, Y_{F_{2}}^{i-1}\right)\right. \\
& \left.-H\left(Y_{1 i}, Y_{F_{1} i}, Y_{F_{2} i} \mid W_{1}, W_{2}, Y_{1}^{i-1}, Y_{F_{1}}^{i-1}, Y_{F_{2}}^{i-1}\right)\right)+n \epsilon_{1}^{(n)} \\
& =\sum_{i=1}^{n}\left(H\left(Y_{1 i}, Y_{F_{1} i}, Y_{F_{2} i} \mid W_{2}, X_{2 i}, Y_{1}^{i-1}, Y_{F_{1}}^{i-1}, Y_{F_{2}}^{i-1}\right)\right. \\
& \left.-H\left(Y_{1 i}, Y_{F_{1} i}, Y_{F_{2} i} \mid X_{2 i}, W_{1}, W_{2}, Y_{1}^{i-1}, Y_{F_{1}}^{i-1}, Y_{F_{2}}^{i-1}\right)\right) \\
& +n \epsilon_{1}^{(n)} \\
& \leq \sum_{i=1}^{n}\left(H\left(Y_{1 i}, Y_{F_{1} i}, Y_{F_{2} i} \mid X_{2 i}, Y_{F_{1}}^{i-1}, Y_{F_{2}}^{i-1}\right)\right. \\
& \left.-H\left(Y_{1 i}, Y_{F_{1} i}, Y_{F_{2} i} \mid X_{2 i}, W_{1}, W_{2}, Y_{1}^{i-1}, Y_{F_{1}}^{i-1}, Y_{F_{2}}^{i-1}\right)\right) \\
& +n \epsilon_{1}^{(n)} \\
& \leq \sum_{i=1}^{n}\left(H\left(Y_{1 i}, Y_{F_{1}}, Y_{F_{2} i} \mid X_{2 i}, Y_{F_{1}}^{i-1}, Y_{F_{2}}^{i-1}\right)\right. \\
& \left.-H\left(Y_{1 i}, Y_{F_{1}}, Y_{F_{2} i} \mid X_{1 i}, X_{2 i}, W_{1}, W_{2}, Y_{1}^{i-1}, Y_{F_{1}}^{i-1}, Y_{F_{2}}^{i-1}\right)\right) \\
& +n \epsilon_{1}^{(n)} \\
& =\sum_{i=1}^{n}\left(H\left(Y_{1 i}, Y_{F_{1} i}, Y_{F_{2} i} \mid X_{2 i}, Y_{F_{1}}^{i-1}, Y_{F_{2}}^{i-1}\right)\right. \\
& \left.-H\left(Y_{1 i}, Y_{F_{1}}, Y_{F_{2} i} \mid X_{1 i}, X_{2 i}, Y_{F_{1}}^{i-1}, Y_{F_{2}}^{i-1}\right)\right)+n \epsilon_{1}^{(n)} \\
& =\sum_{i=1}^{n} I\left(X_{1 i} ; Y_{1 i}, Y_{F_{1} i}, Y_{F_{2} i} \mid X_{2 i}, Y_{F_{1}}^{i-1}, Y_{F_{2}}^{i-1}\right)+n \epsilon_{1}^{(n)} \\
& =n I\left(X_{1} ; Y_{1}, Y_{F_{1}}, Y_{F_{2}} \mid X_{2}, T\right)+n \epsilon_{1}^{(n)}
\end{aligned}
$$




$$
h\left(Y_{F_{1}}, Y_{F_{2}} \mid T=t\right) \geq \frac{1}{2} \log \left((2 \pi \mathrm{e})^{2} \sigma_{Z_{1}}^{2} \sigma_{Z_{2}}^{2}+2 \pi \mathrm{e}\left(\sigma_{Z_{1}}^{2}+\sigma_{Z_{2}}^{2}\right) \mathrm{e}^{2 h(Y \mid T=t)}\right) .
$$

in the first term, (256) follows from the fact that conditioning reduces entropy and by introducing $X_{1 i}$ in the conditioning in the second term and (257) follows from the memoryless property of the channel. Finally, we define $X_{1}=X_{1 Q}, X_{2}=X_{2 Q}$, $T=\left(Q, Y_{F_{1}}^{Q-1}, Y_{F_{2}}^{Q-1}\right), Y_{1}=Y_{1 Q}, Y_{2}=Y_{2 Q}, Y_{F_{1}}=Y_{F_{1} Q}$ and $Y_{F_{2}}=Y_{F_{2} Q}$, where $Q$ is a random variable which is uniformly distributed over $\{1, \ldots, n\}$ and is independent of all other random variables.

Similarly, we have

$$
\begin{aligned}
R_{2} & \leq I\left(X_{2} ; Y_{2}, Y_{F_{1}}, Y_{F_{2}} \mid X_{1}, T\right) \\
R_{1}+R_{2} & \leq I\left(X_{1}, X_{2} ; Y_{1}, Y_{2}, Y_{F_{1}}, Y_{F_{2}} \mid T\right)
\end{aligned}
$$

and we also have from the cut-set bound

$$
\begin{aligned}
R_{1} & \leq I\left(X_{1}, X_{2} ; Y_{1}\right) \\
R_{2} & \leq I\left(X_{2}, X_{2} ; Y_{2}\right) \\
R_{1}+R_{2} & \leq I\left(X_{1}, X_{2} ; Y_{1}, Y_{2}\right)
\end{aligned}
$$

The proof of the dependence balance constraint is along the same lines as in [9] by starting from the inequality $0 \leq I\left(W_{1} ; W_{2} \mid Y_{F_{1}}^{n}, Y_{F_{2}}^{n}\right)-I\left(W_{1} ; W_{2}\right)$ to arrive at

$$
I\left(X_{1} ; X_{2} \mid T\right) \leq I\left(X_{1} ; X_{2} \mid Y_{F_{1}}, Y_{F_{2}}, T\right) .
$$

This completes the Proof of Theorem 2.

\section{Proof of (78)}

In the following derivation of (78), we have dropped conditioning on $T=t$, for the purpose of simplicity. Substituting (80), (81), (82), and (83) in (76), we have

$$
\begin{aligned}
N\left(\Lambda_{1}^{1 / 2} \mathbf{Y}+\mathbf{V}\right) & =N\left(\sqrt{\kappa} Y+V_{1}, V_{2}\right) \\
& =\frac{1}{(2 \pi \mathrm{e})} \mathrm{e}^{h\left(\sqrt{\kappa} Y+V_{1}, V_{2}\right)} \\
& =\frac{1}{\sqrt{(2 \pi \mathrm{e})}} \mathrm{e}^{h\left(\sqrt{\kappa} Y+V_{1}\right)} .
\end{aligned}
$$

We also note the following inequality:

$$
\begin{aligned}
h\left(\sqrt{\kappa} Y+V_{1}\right) & \geq \frac{1}{2} \log \left(\mathrm{e}^{2 h(\sqrt{\kappa} Y)}+2 \pi \mathrm{e}\right) \\
& =\frac{1}{2} \log \left(\kappa \mathrm{e}^{2 h(Y)}+2 \pi \mathrm{e}\right)
\end{aligned}
$$

where (269) follows from the scalar EPI [8] and (270) follows from the fact that for any scalar $c, h(c Y)=h(Y)+\log (|c|)$ [8]. Substituting (270) in (268), we obtain

$$
N\left(\Lambda_{1}^{1 / 2} \mathbf{Y}+\mathbf{V}\right) \geq\left(\frac{\kappa \mathrm{e}^{2 h(Y)}+2 \pi \mathrm{e}}{(2 \pi \mathrm{e})}\right)^{1 / 2}
$$

Similarly, we also have

$$
N\left(\Lambda_{2}^{1 / 2} \mathbf{Y}+\mathbf{V}\right) \geq\left(\frac{\kappa \mathrm{e}^{2 h(Y)}+2 \pi \mathrm{e}}{(2 \pi \mathrm{e})}\right)^{1 / 2}
$$

Therefore, we have

$$
\begin{aligned}
\mu N\left(\Lambda_{1}^{1 / 2} \mathbf{Y}+\mathbf{V}\right)+(1-\mu) & N\left(\Lambda_{2}^{1 / 2} \mathbf{Y}+\mathbf{V}\right) \\
\geq & \left(\frac{\kappa \mathrm{e}^{2 h(Y)}+2 \pi \mathrm{e}}{(2 \pi \mathrm{e})}\right)^{1 / 2}
\end{aligned}
$$

Moreover, the right hand side of (76) simplifies to

$$
\begin{aligned}
& N\left(\left(\mu \Lambda_{1}+(1-\mu) \Lambda_{2}\right)^{1 / 2} \mathbf{Y}+\mathbf{V}\right) \\
& =\frac{1}{(2 \pi \mathrm{e})} \mathrm{e}^{h\left(\sqrt{\mu \kappa Y+V_{1}}, \sqrt{(1-\mu) \kappa} Y+V_{2}\right)} \\
& \left.\left.=\frac{1}{(2 \pi \mathrm{e})} \mathrm{e}^{h\left(( \mu \Lambda _ { 1 } + ( 1 - \mu ) \Lambda _ { 2 } ) ^ { 1 / 2 } \left[Y_{F_{1}}\right.\right.} \quad Y_{F_{2}}\right]^{T}\right) \\
& =\frac{1}{(2 \pi \mathrm{e}) \sqrt{\sigma_{Z_{1}}^{2} \sigma_{Z_{2}}^{2}}} \mathrm{e}^{h\left(Y_{F_{1}}, Y_{F_{2}}\right)} .
\end{aligned}
$$

Using (272)-(276) and substituting in (76), we obtain

$$
\left(\frac{\kappa \mathrm{e}^{2 h(Y)}+2 \pi \mathrm{e}}{(2 \pi \mathrm{e})}\right)^{1 / 2} \leq \frac{1}{(2 \pi \mathrm{e}) \sqrt{\sigma_{Z_{1}}^{2} \sigma_{Z_{2}}^{2}}} \mathrm{e}^{h\left(Y_{F_{1}}, Y_{F_{2}}\right)}
$$

Simplifying (277) by substituting the value of $\kappa$ and reintroducing the conditioning on $T=t$, we have the proof of (78) (see (278) at the top of the page):

\section{ACKNOWLEDGMENT}

The authors would like the thank the anonymous reviewers and the Associate Editor for their detailed and valuable comments which led to the shortening of the proofs of Theorems 2 and 5 .

\section{REFERENCES}

[1] N. Gaarder and J. Wolf, "The capacity region of a multiple-access discrete memoryless channel can increase with feedback," IEEE Trans. Inf. Theory, vol. IT-21, no. 1, pp. 100-102, Jan. 1975. 
[2] A. B. Carleial, "Multiple-access channels with different genaralized feedback signals," IEEE Trans. Inf. Theory, vol. IT-28, no. 6, pp. 841-850, Nov. 1982.

[3] F. M. J. Willems, E. C. van der Meulen, and J. P. M. Schalkwijk, "Achievable rate region for the multiple access channel with generalized feedback," in Proc. Annual Allerton Conf. Communication, Control and Computing, 1983, pp. 284-292.

[4] D. Tuninetti, "On interference channels with generalized feedback," in IEEE Int. Symp. Information Theory, Jun. 2007.

[5] A. Host-Madsen, "Capacity bounds for cooperative diversity," IEEE Trans. Inf. Theory, vol. 52, no. 4, pp. 1522-1544, Apr. 2006.

[6] T. Han and K. Kobayashi, "A new achievable rate region for the interference channel," IEEE Trans. Inf. Theory, vol. IT-27, no. 1, pp. 49-60, Jan. 1981.

[7] I. Sason, "On achievable rate regions for the Gaussian interference channel," IEEE Trans. Inf. Theory, vol. 50, no. 6, pp. 1345-1356, June 2004.

[8] T. M. Cover and J. A. Thomas, Elements of Information Theory. New York: Wiley, 1991.

[9] A. P. Hekstra and F. M. J. Willems, "Dependence balance bounds for single output two-way channels," IEEE Trans. Inf. Theory, vol. 35, no. 1, pp. 44-53, Jan. 1989.

[10] L. Ozarow, "The capacity of the white Gaussian multiple access channel with feedback," IEEE Trans. Inf. Theory, vol. 30, no. 4, pp. 623-629, Jul. 1984.

[11] G. Kramer, "Capacity results for the discrete memoryless network," IEEE Trans. Inf. Theory, vol. 49, no. 1, pp. 4-21, Jan. 2003.

[12] G. Kramer and M. Gastpar, "Dependence balance and the Gaussian multiple access with feedback," in IEEE Information Theory Workshop, Punta del Este, Uruguay, Mar. 13-17, 2006, pp. 198-202.

[13] M. Gastpar and G. Kramer, "On cooperation via noisy feedback," in Int. Zurich Seminar on Communications (IZS), Feb. 2006, pp. 146-149.

[14] A. Sendonaris, E. Erkip, and B. Aazhang, "User cooperation diversity—Part I: System description," IEEE Trans. Commun., vol. 51, no. 11, pp. 1927-1938, Nov. 2003.

[15] M. Gastpar and G. Kramer, "On noisy feedback for interference channels," in Proc. Asilomar Conf. on Signals, Systems, and Computers, Pacific Grove, CA, USA, Oct. 29-Nov. 12006.

[16] M. Payaro and D. Palomar, "A multivariate generalization of Costa's entropy power inequality," in IEEE Int. Symp. Information Theory, Jul. 2008.

[17] M. H. M. Costa, "A new entropy power inequality," IEEE Trans. Inf. Theory, vol. IT-31, no. 6, pp. 751-760, Nov. 1985.

[18] S. I. Bross, A. Lapidoth, and M. A. Wigger, "The Gaussian MAC with conferencing encoders," in IEEE Int. Symp. Information Theory, July 2008.

[19] V. Venkatesan, "Optimality of Gaussian Inputs for a Multi-Access Achievable Rate Region," Semester thesis, ETH Zurich, Switzerland, Jun. 2007.

[20] M. A. Wigger, "Cooperation on the Multiple-Access Channel," Ph.D thesis, ETH Zurich, Switzerland, 2008.

[21] M. A. Wigger and G. Kramer, "Three-user MIMO MACs with cooperation," in IEEE Information Theory Workshop, Volos, Greece, Jun. 2009.
[22] D. Tuninetti, "An outer bound region for interference channels with generalized feedback," in Information Theory and Applications Workshop, San Diego, Jan. 2010.

[23] V. Prabhakaran and P. Viswanath, "Interference channels with source cooperation," IEEE Trans. Inf. Theory, vol. 57, no. 5, pp. 2781-2804, May 2011.

Ravi Tandon (S'03-M'09) received the B.Tech. degree in electrical engineering from the Indian Institute of Technology, Kanpur, India, in 2004 and the Ph.D. degree from the Department of Electrical and Computer Engineering, University of Maryland, College Park in June, 2010.

Since July 2010, he is a post-doctoral research fellow at the Department of Electrical Engineering at Princeton University. His research interests include network information theory and wireless communication theory.

Sennur Ulukus (S'90-M'98) received the B.S. and M.S. degrees in electrical and electronics engineering from Bilkent University, Ankara, Turkey, in 1991 and 1993, respectively, and the Ph.D. degree in electrical and computer engineering from Rutgers University, NJ, in 1998. During her Ph.D. studies, she was with the Wireless Information Network Laboratory (WINLAB), Rutgers University.

From 1998 to 2001, she was a Senior Technical Staff Member at AT\&T Labs-Research. In 2001, she joined the University of Maryland at College Park, where she is currently an Associate Professor in the Department of Electrical and Computer Engineering, with a joint appointment at the Institute for Systems Research (ISR). Her research interests are in wireless communication theory and networking, network information theory for wireless networks, signal processing for wireless communications and security for multiuser wireless communications.

Dr. Ulukus is a recipient of the 2005 NSF CAREER Award, and a co-recipient of the 2003 IEEE Marconi Prize Paper Award in Wireless Communications. She serves/served as an Associate Editor for the IEEE Transactions on Information Theory since 2007, as an Associate Editor for the IEEE TRANSACTIONS ON COMMUNICATIONS between 2003-2007, as a Guest Editor for the IEEE TRANSACTIONS ON INFORMATION THEORY for the special issue on interference networks, as a Guest Editor for the IEEE JOURNAL ON SELECTED AREAS IN COMMUNICATIONS for the special issue on multiuser detection for advanced communication systems and networks, as the co-chair of the Communication Theory Symposium at the 2007 IEEE Global Telecommunications Conference, as the co-chair of the Medium Access Control (MAC) Track at the 2008 IEEE Wireless Communications and Networking Conference, as the co-chair of the Wireless Communications Symposium at the 2010 IEEE International Conference on Communications, as the co-chair of the 2011 Communication Theory Workshop, as the co-chair of the Physical-Layer Security Workshop at the 2011 IEEE International Conference on Communications, and as the Secretary of the IEEE Communication Theory Technical Committee (CTTC) in 2007-2009. 